\title{
UNIQUENESS OF CONSTRAINED VISCOSITY SOLUTIONS IN HYBRID CONTROL SYSTEMS*
}

\begin{abstract}
MINYI HUANG ${ }^{\dagger}$
Abstract. We study constrained viscosity solutions with an unbounded growth for a class of first order Hamilton-Jacobi-Bellman equations arising in hybrid control systems. To deal with the boundary constraint and rapid growth of the solutions, we construct a particular set of test functions and under very mild conditions establish a comparison theorem which gives the estimate of distance between the subsolution and the supersolution. The comparison theorem implies uniqueness of the constrained viscosity solution if its existence is ensured; and under some additional assumptions we give an existence result by showing that the value function is a constrained viscosity solution. We then apply the obtained uniqueness results to an optimal scheduling problem and finally to stochastic manufacturing systems.
\end{abstract}

Key words. hybrid control systems, optimal control, HJB equations, constrained viscosity solutions, fluid models, manufacturing systems

AMS subject classifications. 93E20, 93E03, 49L25, 49L20

DOI. $10.1137 / 050635845$

1. Introduction. This paper is concerned with the analysis of a class of first order Hamilton-Jacobi-Bellman (HJB) equations with discrete transitions and state constraints. Such equations arise naturally in the optimal control of stochastic systems with random structural changes in dynamics, which are modeled as Markovian jumps. These systems involve both continuum and discrete components in their evolution and are referred to as hybrid systems, and they have been investigated from a wide range of backgrounds including production planning subject to random machine breakdown and repair [20] and the control of fluid queueing models for communication networks $[19,7,17]$, among others $[6,9,14,24,28]$. Due to physical limitation, a typical feature for many control models is that the system state is restricted to a certain set; for instance, the level for buffers must be maintained nonnegative [20, 25]. To deal with the resulting HJB equation, one needs to take into account both the discrete transitions and the state space constraints and to adopt the notion of appropriately defined constrained viscosity solutions first introduced in [22].

Specifically, Soner studied an optimal control problem and introduced first order constrained viscosity solutions in [22], where the deterministic state trajectory is restricted to a given subset of $\mathbb{R}^{n}$, and in a companion work [23] along this line, viscosity solutions were analyzed for controlled piecewise deterministic Markov processes [6] defined on a subset of $\mathbb{R}$, which leads to an integral HJB equation. Later on, the result in [22] was generalized in [18] by identifying weaker sufficient conditions for ensuring continuity of the value function and in [12] by an additional boundary characterization of the subsolution via a so-called inward Hamiltonian reflecting boundary constraints.

* Received by the editors July 12, 2005; accepted for publication (in revised form) December 27, 2006; published electronically April 13, 2007. This work was partially supported by the Australian Research Council.

http://www.siam.org/journals/sicon/46-1/63584.html

${ }^{\dagger}$ Department of Electrical and Electronic Engineering, The University of Melbourne, Parkville, Victoria 3010, Australia. Current address: Department of Information Engineering, Research School of Information Sciences and Engineering, The Australian National University, Canberra, ACT 0200, Australia (minyi.huang@rsise.anu.edu.au). 


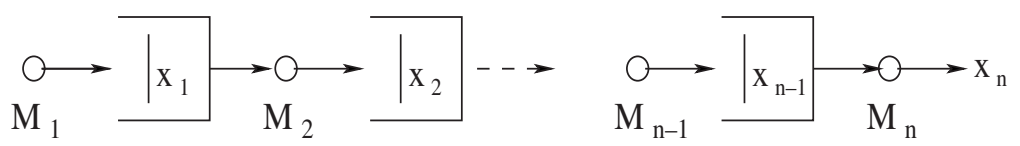

(a)

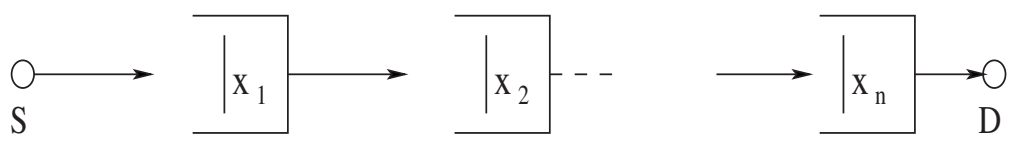

(b)

FIG. 1. (a) A manufacturing system with $n$ machines $\left(M_{i}, 1 \leq i \leq n\right)$ and $n-1$ buffers; (b) a multihop communication network where the source $S$ and destination $D$ are connected by $n$ buffers.

For HJB equations involving finite state Markov chains, viscosity solutions have been studied in $[20,8,26,27]$. In particular, the authors in $[26,27]$ considered controlled random transitions but there were no state constraints.

Although viscosity solutions with state constraints are of importance and have their primary motivation in optimal control, in many application problems, the existing results face limitation. Notably, in the sequence of work $[22,18,12]$ considering first order HJB equations for deterministic systems, uniqueness is obtained for uniformly continuous and bounded solutions. In [15] and [5], bounded continuous solutions were analyzed on a bounded domain. Also, in a singular perturbation control problem with partial state constraints [1], uniqueness and existence theorems were established with bounded continuous solutions. For illustrating the limitation of those previous results, we consider the optimal control of a single buffer fluid model with controlled input and output and, as a well-motivated practice, introduce a linear holding cost for a positive buffer level (see section 6 for details). This readily leads to unbounded value functions, and existing results for constrained viscosity solutions are difficult to apply.

In this work we study uniqueness of constrained viscosity solutions for a class of stochastic hybrid systems. We concentrate our attention to two concrete types of domains for the state variable. The particular structure of the state space has adequate generality and is frequently encountered in a wide range of application problems arising in manufacturing systems and communication networks [20, 25, 19] (see Figure 1 for illustration), though a generalization of the state space to other forms is possible. In introducing our solution notion, we generalize the definition of constrained viscosity solutions for standard HJB equations of deterministic models to a coupled HJB equation system. Resulting from the state space constraints, this definition leads to specifying the viscosity sub/supersolution in two different regions, respectively, i.e., characterizing the subsolution in a smaller region - the interior of the constrained state space. Such a differentiation by two regions is important for developing a solution framework for uniqueness analysis. We prove uniqueness of the solution within the class of functions satisfying a polynomial growth and local Hölder continuity. In establishing the comparison result in this paper, a crucial step is to obtain suitable test functions involved in the definition of constrained viscosity solutions. Towards this end, we construct the auxiliary function $\Phi$ by first dominating the sub/supersolution growth by an exponential function and then introducing a pair of perturbation parameters $(\tau, \varepsilon)$ [see (11)] such that the resulting maxima (w.r.t. $x$ ) can be tuned to the interior of the state space to generate desired test functions for the subsolution. 
The proof of the comparison theorem depends on generalizing typical techniques for deterministic systems $[22,2]$.

For proving existence results in the general model, a quite difficult step is to show continuity of the value function. The key idea in our analysis is to truncate a small time interval by the jump time of the Markov chain so that locally the system dynamics act like a time-invariant model. This resulting feature enables us to use a certain time-shifting technique to construct auxiliary admissible controls for cost estimates. In particular, using a recursive estimation procedure, we obtain Hölder continuity of the value function, and we mention that as a byproduct this method can be used to strengthen some existing continuity results in the literature for state constrained optimal control problems.

Our work differs from most existing analysis on constrained viscosity solutions for deterministic systems in that we need to deal with a system of coupled equations and the solution growth is rapid. Our solution notion for the coupled HJB equation system and the uniqueness results provide a unified analytical basis for the optimal control of this class of hybrid systems. In particular, our uniqueness results are applicable to classical stochastic manufacturing models (see, e.g., [20]), where to our best knowledge the existing work has not provided uniqueness results for the coupled HJB equations when nonnegative buffer level constraints are imposed.

The organization of the paper is as follows. In section 2, we first describe the optimal control problem for the hybrid system and introduce the notion of constrained viscosity solutions. The comparison result and uniqueness theorem are stated in section 3. The proof of the comparison theorem is technical and postponed to section 4. For the general hybrid system model, section 5 first shows Hölder continuity of the value function under some technical conditions and proves that it is the unique constrained viscosity solution. In section 6 , we study an optimal data traffic scheduling problem and prove the existence and uniqueness of constrained viscosity solutions by applying the result in section 3. In section 7, we further apply the results in section 3 to a well-studied stochastic manufacturing system, which complements existence theorems in the manufacturing literature [20]. Finally, a few concluding remarks are presented in section 8 .

2. The HJB equation and constrained viscosity solutions. Consider a hybrid control system described by the following differential equation:

$$
\frac{d X(t)}{d t}=F(X(t), \theta(t), u(t)), \quad t \geq 0
$$

with initial condition $X(0) \in \bar{Q}$. Here $X$ and $\theta$ are called the state and mode variables, respectively. The trajectory of $X$ on $[0, \infty)$ is required to be in $\bar{Q}$, which is a closed subset of $\mathbb{R}^{n}$ with a nonempty interior $Q$. Moreover, $\theta$ is a continuous time Markov chain with state space $\Theta=\{1,2, \ldots, m\}$ and transition probability rate matrix $\Pi_{\theta}=$ $\left(\pi_{i j}\right)_{m \times m}$, which is also called the generator. It is assumed that, with probability one, the trajectory of $\theta$ is right continuous with left limit. Given $\theta(t)=k$, the control $u(t)$ takes values from a compact set $U_{k} \subset \mathbb{R}^{d}$. Let $\mathcal{F}_{t}$ denote the $\sigma$-algebra generated by the Markov chain $\theta$ up to time $t$, i.e., $\mathcal{F}_{t}=\sigma(\theta(s), s \leq t)$. Associated with $X(0)=x$ and $\theta(0)=k$, the admissible control set is written as $\mathcal{U}_{x, k}$ consisting of all controls $u(\cdot)$ satisfying $u(t) \in U_{\theta(t)}$ and adapted to $\mathcal{F}_{t}$ such that $P\{X(t) \in \bar{Q}, \forall t \geq 0\}=1$. We make the convention that for all $(x, k) \in \bar{Q} \times \Theta, \mathcal{U}_{x, k}$ is nonempty and that the state process $X(t)$ associated with an admissible control is uniquely determined on $[0, \infty)$ with exception on a null set of samples. Given initial condition $(x, k) \in \bar{Q} \times \Theta$ 
at $t=0$, let the cost function be given by

$$
\begin{aligned}
v(x, k) & =\inf _{u \in \mathcal{U}_{x, k}} J(x, k, u) \\
& \triangleq \inf _{u \in \mathcal{U}_{x, k}} E\left[\int_{0}^{\infty} e^{-\rho t} L(X(t), \theta(t), u(t)) d t \mid X(0)=x, \theta(0)=k\right],
\end{aligned}
$$

where $\rho>0$ is a discount factor and $L$ is the cost integrand before discount.

To facilitate the subsequent analysis, we set some convention on notation. We may alternatively denote $X(t)$ as $X_{t}$ with a real-valued subscript $t \geq 0$, and the same convention holds for $u(t)$ and $\theta(t)$, etc. The letter $u$ may stand for a value in $U_{k}$ for a certain $k \in \Theta$ or a control adapted to $\mathcal{F}_{t}$; the specific interpretation should be clear from the context. Throughout the paper, for a real-valued vector $y,|y|$ denotes its Euclidean norm.

For any function $\varphi: \Theta \rightarrow \mathbb{R}$, we define the map

$$
\left[\Pi_{\theta} \varphi(\cdot)\right](i)=\sum_{j \neq i} \pi_{i j}[\varphi(j)-\varphi(i)]
$$

where $\pi_{i i}+\sum_{j \neq i} \pi_{i j}=0$.

We assume for any given $k \in \Theta$, both $F(x, k, u)$ and $L(x, k, u)$ are continuous in $(x, u) \in \bar{Q} \times U_{k}$. A formal application of dynamic programming leads to the following equation system:

$$
\rho v(x, k)=\inf _{u \in U_{k}}\left[v_{x}^{T}(x, k) F(x, k, u)+\left[\Pi_{\theta} v(x, \cdot)\right](k)+L(x, k, u)\right],
$$

where $(x, k) \in \bar{Q} \times \Theta$ and the superscript $(\cdot)^{T}$ denotes the transpose of a vector or matrix. Note that due to the action of the generator, (4) gives a system of $m$ coupled equations. For convenience of exposition, we simply refer to (4) as the HJB equation for the underlying optimal control problem. Write

$$
\tilde{H}\left(x, k, v_{x}(x, k), v(x, \cdot), u\right)=v_{x}^{T}(x, k) F(x, k, u)+\left[\Pi_{\theta} v(x, \cdot)\right](k)+L(x, k, u) .
$$

Then the HJB equation (4) may be written in the compact form:

$$
\begin{aligned}
\rho v(x, k) & =\inf _{u \in U_{k}} \tilde{H}\left(x, k, v_{x}(x, k), v(x, \cdot), u\right) \\
& \triangleq H\left(x, k, v_{x}(x, k), v(x, \cdot)\right), \quad(x, k) \in \bar{Q} \times \Theta,
\end{aligned}
$$

where the dot entry in (5) indicates that for each fixed $k$, the term $H$ depends on the whole vector $[v(x, 1), \ldots, v(x, m)]$.

DeFinition 1. Let $\underline{v}(x, k), \bar{v}(x, k)$, and $v(x, k)$ be functions from $\bar{Q} \times \Theta$ to $\mathbb{R}$, each being continuous in $x$ for all $k \in \Theta$.

(i) $\underline{v}(x, k)$ is a viscosity subsolution to (5) on $Q \times \Theta$ if for any $k_{0} \in \Theta$ and any function $\phi \in C^{1}(\bar{Q})$, we have

$$
\rho \underline{v}\left(x_{0}, k_{0}\right)-H\left(x_{0}, k_{0}, \phi_{x}\left(x_{0}\right), \underline{v}\left(x_{0}, \cdot\right)\right) \leq 0
$$

at $x_{0}$, whenever $\underline{v}\left(x, k_{0}\right)-\phi(x)$ attains a local maximum at $x=x_{0} \in Q$.

(ii) $\bar{v}(x, k)$ is a viscosity supersolution to (5) on $\bar{Q} \times \Theta$ if for any $k_{0} \in \Theta$ and $\phi \in C^{1}(\bar{Q})$, we have

$$
\rho \bar{v}\left(x_{0}, k_{0}\right)-H\left(x_{0}, k_{0}, \phi_{x}\left(x_{0}\right), \bar{v}\left(x_{0}, \cdot\right)\right) \geq 0
$$

at $x_{0}$, whenever $\bar{v}\left(x, k_{0}\right)-\phi(x)$ attains a local minimum at $x=x_{0} \in \bar{Q}$. 
(iii) $v(k, x)$ is called a constrained viscosity solution on $\bar{Q} \times \Theta$ to (5) if it is both a viscosity subsolution on $Q \times \Theta$ and a viscosity supersolution on $\bar{Q} \times \Theta$.

In the definition of the viscosity supersolution, the minima $x_{0}$ may lie on the boundary of $\bar{Q}$. The function $\phi$ involved in either (i) or (ii) in Definition 1 is called the test function.

Denote by $C_{p}(\bar{Q} \times \Theta)$ the set of functions $g(x, k)$ from $\bar{Q} \times \Theta$ to $\mathbb{R}$, which are continuous in $x \in \bar{Q}$ for any given $k \in \Theta$ and have a polynomial growth rate, i.e., for any $g \in C_{p}(\bar{Q} \times \Theta)$, one can find positive constants $C$ and $b$, depending on that particular function, such that $|g(x, k)| \leq C\left(1+|x|^{b}\right)$ for all $(x, k) \in \bar{Q} \times \Theta$. For $a_{1}, a_{2} \in \mathbb{R}$, denote $a_{1} \vee a_{2}=\max \left\{a_{1}, a_{2}\right\}$, and $a_{1} \wedge a_{2}=\min \left\{a_{1}, a_{2}\right\}$. Given $\gamma \in(0,1]$ and $g \in C_{p}(\bar{Q} \times \Theta)$, define

$$
\operatorname{Hol}(g, \gamma, R) \triangleq \sup _{k \in \Theta} \sup _{|x| \vee\left|x^{\prime}\right| \leq R} \frac{\left|g\left(k, x^{\prime}\right)-g(k, x)\right|}{\left|x^{\prime}-x\right|^{\gamma}},
$$

where $x, x^{\prime} \in \bar{Q}$ and $0<R<\infty$. The value $\operatorname{Hol}(g, \gamma, R) \leq \infty$ is called the local Hölder constant for $g$ associated with $R>0$, where $\gamma$ is the Hölder exponent. For the case $\gamma=1, \operatorname{Hol}(g, 1, R)$ reduces to the local Lipschitz constant and is denoted as $\operatorname{Lip}(g, R)$.

Define $C_{p, H o l}^{l o c}(\bar{Q} \times \Theta)$ as the class of functions $g \in C_{p}(\bar{Q} \times \Theta)$ satisfying local Hölder continuity; i.e., there exists a Hölder exponent $\gamma \in(0,1]$ such that $\operatorname{Hol}(g, \gamma, R)<\infty$ for all $R>0$. Furthermore, we define $C_{p, L i p}^{\text {loc }}(\bar{Q} \times \Theta)$ as the class of functions $g \in$ $C_{p}(\bar{Q} \times \Theta)$ satisfying local Lipschitz continuity in $x$; i.e., $\operatorname{Lip}(g, R)<\infty$ for all $R>0$.

Obviously, $C_{p, L i p}^{\text {loc }}(\bar{Q} \times \Theta) \subset C_{p, H o l}^{l o c}(\bar{Q} \times \Theta)$. In addition, if $g \in C_{p, H o l}^{l o c}(\bar{Q} \times \Theta)$ with Hölder exponent $\gamma_{2}$ and $0<\gamma_{1}<\gamma_{2} \leq 1, g$ is also locally Hölder continuous with exponent $\gamma_{1}$.

In establishing our main results, we concentrate on two types of structures for $\bar{Q}$.

Case (i). For state constraint in a subspace:

$$
\bar{Q}_{a} \triangleq[0, \infty)^{n-1} \times(-\infty, \infty),
$$

where the integer $n \geq 2$. The interior of the set is $Q_{a}=(0, \infty)^{n-1} \times(-\infty, \infty)$.

Case (ii). For state constraint in full space:

$$
\bar{Q}_{b} \triangleq[0, \infty)^{n},
$$

where $n \geq 1$. The interior of the set is $Q_{b}=(0, \infty)^{n}$.

Corresponding to $\bar{Q}_{a}$ and $\bar{Q}_{b}$, the state variable $x$ is restricted to the positive orthant of $\mathbb{R}^{n}$ or its $n$-1-dimensional subspace. Indeed, cases (i) and (ii) can cover fairly general application models as shown in Figure 1, and they are also applicable to systems with more complicated buffer interconnection; see, e.g., [21]. It is worth noting that in the manufacturing fluid model given by Figure 1(a), the first $n-1$ entries in $x$ correspond to buffer levels and must be positive; the last entry $x_{n}$, which denotes the inventory level of the final product, however, can be negative and interpreted as backlog. Although our technique developed in this paper may be extended to deal with other forms of $\bar{Q}$, we do not intend to treat the most general form.

3. The comparison theorem and uniqueness of solutions. The objective of this section is to establish a comparison result which plays an important role in proving uniqueness. Existence analysis will be presented for the general model in section 5 and for more concrete models in sections 6 and 7 . 
Let $L_{i}(x, k, u), i=1,2$, be two functions with $u \in U_{k}$ and $(x, k) \in \bar{Q} \times \Theta$. Replacing $L(x, k, u)$ by $L_{i}(x, k, u)$ in the original HJB equation (4), we write two new equations:

$$
\begin{aligned}
& \rho v(x, k)=H_{1}\left(x, k, v_{x}(x, k), v(x, \cdot)\right), \\
& \rho v(x, k)=H_{2}\left(x, k, v_{x}(x, k), v(x, \cdot)\right),
\end{aligned}
$$

where $(x, k) \in \bar{Q} \times \Theta$ and the construction for $H_{i}, i=1,2$, is obvious.

3.1. Main results. We make the following assumptions.

(A1) For any given $k \in \Theta, F(x, k, u)$ and $L_{i}(x, k, u), i=1,2$, are continuous in $(x, u) \in \bar{Q} \times U_{k}$.

$\left(\mathrm{A} 1^{\prime}\right)$ For any given $k \in \Theta, F(x, k, u)$ and $L(x, k, u)$ are continuous in $(x, u) \in$ $\bar{Q} \times U_{k}$.

(A2) $F_{\max } \triangleq \sup _{(x, k) \in \bar{Q} \times \Theta} \sup _{u \in U_{k}}|F(x, k, u)|<\infty$.

Under (A1) and $\left(\mathrm{A} 1^{\prime}\right)$, we have the following equicontinuity in $x$ on compact sets. Let $\varphi$ stand for $F, L$, or $L_{i}$. For a given compact subset $B_{\bar{Q}}$ of $\bar{Q}$, when $x, x^{\prime} \in B_{\bar{Q}}$ and $\left|x-x^{\prime}\right| \rightarrow 0$, we have

$$
\left|\varphi(x, k, u)-\varphi\left(x^{\prime}, k, u\right)\right| \rightarrow 0
$$

with a vanishing rate not depending on $(k, u)$.

THEOREM 2. Let $\bar{Q}$ be either $\bar{Q}_{a}$ or $\bar{Q}_{b}$, and suppose (A1)-(A2) hold. If $v_{1}$, $v_{2} \in C_{p, H o l}^{l o c}(\bar{Q} \times \Theta)$ are, respectively, a viscosity subsolution to (8) on $Q \times \Theta$ and a viscosity supersolution to (9) on $\bar{Q} \times \Theta$, then the inequality holds:

$$
\sup _{\bar{Q} \times \Theta}\left[v_{1}(x, k)-v_{2}(x, k)\right] \leq \rho^{-1} \sup _{\bar{Q} \times \Theta U_{k}}\left[L_{1}(x, k, u)-L_{2}(x, k, u)\right] .
$$

Theorem 2 is the so-called comparison theorem, and it immediately implies the following uniqueness theorem.

THEOREM 3. Let $\bar{Q}$ be either $\bar{Q}_{a}$ or $\bar{Q}_{b}$, and suppose $\left(\mathrm{A}^{\prime}\right)$ and (A2) hold. If $v \in C_{p, \mathrm{Hol}}^{\text {loc }}(\bar{Q} \times \Theta)$ is a constrained viscosity solution to (5), then it is unique in the function class $C_{p, H o l}^{\text {loc }}(\bar{Q} \times \Theta)$.

3.2. Some preliminary lemmas. To prove Theorem 2 , we need to establish a sequence of preliminary results. The basic approach is to introduce a suitable comparison function $\Phi$ for the construction of smooth test functions $\phi$ to generate the local minima and maxima and then to apply the definition of viscosity sub/supersolutions. A key technique will be developed such that the obtained maxima for $v_{1}-\phi$, as specified during the proof of Theorem 2, do not occur at the boundary of $\bar{Q}$, which is crucial for subsequently applying the definition of viscosity subsolutions.

Let $v_{1}$ and $v_{2}$ be the viscosity sub/supersolution, respectively. For both Case (i) $\bar{Q}=\bar{Q}_{a}=[0, \infty)^{n-1} \times(-\infty, \infty)$ and Case (ii) $\bar{Q}=\bar{Q}_{b}=[0, \infty)^{n}$, we use the same function $\Phi(x, y, k)$ constructed as follows. Denote $\mathbf{1}_{n}=(1,1, \ldots, 1)^{T}$, and for $v_{1}, v_{2} \in C_{p, H o l}^{l o c}(\bar{Q} \times \Theta)$, let

$$
\begin{aligned}
\Phi(x, y, k)= & v_{1}(x, k)-v_{2}(y, k)-\left|\frac{x-y}{\varepsilon}-\tau \mathbf{1}_{n}\right|^{2} \\
& -\alpha[\zeta(x)+\zeta(y)], \quad x, y \in \bar{Q},
\end{aligned}
$$

where $\zeta(x)=\exp \left(\beta \sqrt{|x|^{2}+1}\right)$, with $\beta=\rho F_{\max }^{-1}$, and $\varepsilon, \tau, \alpha$ are all parameters chosen within the interval $(0,1]$ throughout sections 3 and 4 . 
The construction of $\Phi$ is based on the methods in $[20,22,11,5]$; however, with the simultaneous appearance of state constraints and rapid growth, it is necessary to predominate $v_{1}$ and $v_{2}$ by the exponential term $\zeta(x)$ and subsequently insert the small perturbation term $\tau \mathbf{1}_{n}$, the magnitude of which can be adjusted independently. This differs from the technique in $[22,5]$. During the maximization of $\Phi, \tau$ causes a useful asymmetry between $x$ and $y$ in producing the increment of $\Phi$. Such an effect is further amplified by reducing $\varepsilon$ provided that $\tau$ is fixed first, and this ensures that $x$ can be tuned to the interior of $\bar{Q}$ leading to desired test functions.

Since both $v_{1}$ and $v_{2}$ have a polynomial growth rate, it is clear that there exists $(\hat{x}, \hat{y}, \hat{k}) \in \bar{Q} \times \bar{Q} \times \Theta \triangleq \Gamma$ such that

$$
\Phi(\hat{x}, \hat{y}, \hat{k})=\sup _{(x, y, k) \in \Gamma} \Phi(x, y, k)
$$

where the values of $\hat{x}, \hat{y}$, and $\hat{k}$ depend on $\varepsilon, \tau$ and $\alpha$. However, for a given $\alpha \in(0,1]$, we may obtain a uniform bound for $|\hat{x}|$ and $|\hat{y}|$ when the value of $\varepsilon$ and $\tau$ varies on $(0,1]$.

Lemma 4. Suppose $\bar{Q}=\bar{Q}_{a}$ or $\bar{Q}_{b}$. Let $v_{1}, v_{2} \in C_{p, H o l}^{\text {loc }}(\bar{Q} \times \Theta)$ be given in (11) and $(\hat{x}, \hat{y}, \hat{k})$ be obtained from (12). Then there exists a positive constant, depending only on $\alpha$ and denoted as $C_{\alpha}$, such that

$$
|\hat{x}| \vee|\hat{y}| \leq C_{\alpha} .
$$

Proof. It suffices to analyze for $\bar{Q}=\bar{Q}_{a}$. Since $\Phi(\hat{x}, \hat{y}, \hat{k}) \geq \Phi(0,0, \hat{k})$, it follows that

$$
\begin{aligned}
& v_{1}(\hat{x}, \hat{k})-v_{2}(\hat{y}, \hat{k})-\left|\frac{\hat{x}-\hat{y}}{\varepsilon}-\tau \mathbf{1}_{n}\right|^{2}-\alpha[\zeta(\hat{x})+\zeta(\hat{y})] \\
\geq & v_{1}(0, \hat{k})-v_{2}(0, \hat{k})-n \tau^{2}-\alpha[\zeta(0)+\zeta(0)],
\end{aligned}
$$

which gives

$$
\begin{aligned}
& \alpha[\zeta(\hat{x})+\zeta(\hat{y})]+\left|\frac{\hat{x}-\hat{y}}{\varepsilon}-\tau \mathbf{1}_{n}\right|^{2} \\
\leq & v_{1}(\hat{x}, \hat{k})-v_{1}(0, \hat{k})-v_{2}(\hat{y}, \hat{k})+v_{2}(0, \hat{k})+n \tau^{2}+\alpha[\zeta(0)+\zeta(0)] .
\end{aligned}
$$

Without loss of generality, assume $C_{0}>0$ and $b_{0}>0$ have been found such that $\left|v_{1}(x, k)\right| \vee\left|v_{2}(x, k)\right| \leq C_{0}\left(1+|x|^{b_{0}}\right)$, for $(x, k) \in \bar{Q} \times \Theta$. Since

$$
\alpha[\zeta(\hat{x})+\zeta(\hat{y})] \leq C_{0}\left(4+|\hat{x}|^{b_{0}}+|\hat{y}|^{b_{0}}\right)+n+[\zeta(0)+\zeta(0)],
$$

there exists $C_{\alpha}>0$, depending on $\alpha$ but not on $\varepsilon$ and $\tau$, such that $|\hat{x}| \vee|\hat{y}|$ $\leq C_{\alpha}$.

Notice that the selection of $C_{\alpha}$ implicitly depends on the associated parameters $C_{0}$ and $b_{0}$. However, for convenience of presentation, in our analysis we simply say it depends only on $\alpha$, since $v_{1}$ and $v_{2}$ are assumed to be picked out from $C_{p, H o l}^{l o c}(\bar{Q} \times \Theta)$ and hence fixed.

Lemma 5. Suppose $\bar{Q}=\bar{Q}_{a}$ or $\bar{Q}_{b}$ and fix $\alpha \in(0,1]$. For $(\hat{x}, \hat{y}, \hat{k})$ given in (12), the following properties hold: (i) $\sup _{\varepsilon \in(0,1]} \varepsilon^{-1}|\hat{x}-\hat{y}|=O(1)$, where the right-hand side is independent of $\tau$, and (ii) $\lim _{\varepsilon \rightarrow 0+}|\hat{x}-\hat{y}|=0$ uniformly w.r.t. $\tau$. 
Proof. It is adequate to consider $\bar{Q}=\bar{Q}_{a}$. Since $2 \Phi(\hat{x}, \hat{y}, \hat{k}) \geq \Phi(\hat{x}, \hat{x}, \hat{k})+$ $\Phi(\hat{y}, \hat{y}, \hat{k})$, we get

$$
\begin{aligned}
& 2 v_{1}(\hat{x}, \hat{k})-2 v_{2}(\hat{y}, \hat{k})-2\left|\frac{\hat{x}-\hat{y}}{\varepsilon}-\tau \mathbf{1}_{n}\right|^{2}-2 \alpha[\zeta(\hat{x})+\zeta(\hat{y})] \\
\geq & v_{1}(\hat{x}, \hat{k})-v_{2}(\hat{x}, \hat{k})-n \tau^{2}-2 \alpha \zeta(\hat{x})+v_{1}(\hat{y}, \hat{k})-v_{2}(\hat{y}, \hat{k})-n \tau^{2}-2 \alpha \zeta(\hat{y}) .
\end{aligned}
$$

Suppose $v_{1}, v_{2}$ have exponent $\gamma_{1}, \gamma_{2} \in(0,1]$, respectively, for local Hölder continuity. Hence

$$
\begin{aligned}
\left|\frac{\hat{x}-\hat{y}}{\varepsilon}-\tau \mathbf{1}_{n}\right|^{2} & \leq \frac{1}{2}\left[v_{1}(\hat{x}, \hat{k})-v_{1}(\hat{y}, \hat{k})+v_{2}(\hat{x}, \hat{k})-v_{2}(\hat{y}, \hat{k})\right]+n \tau^{2} \\
& \leq \frac{1}{2}\left[\operatorname{Hol}\left(v_{1}, \gamma_{1}, C_{\alpha}\right)|\hat{x}-\hat{y}|^{\gamma_{1}}+\operatorname{Hol}\left(v_{2}, \gamma_{2}, C_{\alpha}\right)|\hat{x}-\hat{y}|^{\gamma_{2}}\right]+n \tau^{2} \\
& \leq\left(1+C_{\alpha}\right)\left[\operatorname{Hol}\left(v_{1}, \gamma_{1}, C_{\alpha}\right)+\operatorname{Hol}\left(v_{2}, \gamma_{2}, C_{\alpha}\right)\right]+n \tau^{2},
\end{aligned}
$$

since $\hat{x} \vee \hat{y} \leq C_{\alpha}$ by Lemma 4 .

By use of the triangular inequality for norms, for $\tau \in(0,1]$, we get

$$
\begin{aligned}
\left|\frac{\hat{x}-\hat{y}}{\varepsilon}\right| & \leq\left|\frac{\hat{x}-\hat{y}}{\varepsilon}-\tau \mathbf{1}_{n}\right|+\left|\tau \mathbf{1}_{n}\right| \\
& \leq \sqrt{\left(1+C_{\alpha}\right)\left[\operatorname{Hol}\left(v_{1}, \gamma_{1}, C_{\alpha}\right)+\operatorname{Hol}\left(v_{2}, \gamma_{2}, C_{\alpha}\right)\right]+n \tau^{2}}+n \tau \\
& \leq \sqrt{\left(1+C_{\alpha}\right)\left[\operatorname{Hol}\left(v_{1}, \gamma_{1}, C_{\alpha}\right)+\operatorname{Hol}\left(v_{2}, \gamma_{2}, C_{\alpha}\right)\right]+n}+n,
\end{aligned}
$$

which implies assertion (i) and subsequently (ii). This completes the proof.

The proof of Lemmas 4 and 5 adopts the techniques in $[11,20]$ dealing with unbounded viscosity solutions for first order HJB equations. The next lemma is essential for deriving the comparison result in section 4.

Lemma 6. Let $(\hat{x}, \hat{y}, \hat{k})$ be given by $(12)$ and $\hat{x}=\left[\hat{x}_{1}, \ldots, \hat{x}_{n}\right]^{T}, \hat{y}=\left[\hat{y}_{1}, \ldots, \hat{y}_{n}\right]^{T}$. For given $\tau, \alpha \in(0,1]$, if $\varepsilon>0$ is sufficiently small, we have (i) $\hat{x}_{i}>\hat{y}_{i}$ for $1 \leq i \leq$ $n-1$, if $\bar{Q}=\bar{Q}_{a}$, or (ii) $\hat{x}_{i}>\hat{y}_{i}$ for $1 \leq i \leq n$, if $\bar{Q}=\bar{Q}_{b}$, which further implies $\hat{x} \in Q$ for both cases.

Proof. We give only the proof for assertion (i). The proof for assertion (ii) can be handled similarly. The proof is quite technical, and we break it into three steps.

Step 1. Let $\alpha$ and $\tau$ be given with $\bar{Q}=\bar{Q}_{a}$. We assume assertion (i) is invalid, and hence there exists a sequence $\varepsilon_{i} \downarrow 0, i \geq 1$, such that there is at least one (denoted as the $n_{i}$ th) coordinate component satisfying

$$
\hat{x}_{n_{i}}^{(i)}-\hat{y}_{n_{i}}^{(i)} \leq 0,
$$

where $\left(\hat{x}^{(i)}, \hat{y}^{(i)}, \hat{k}^{(i)}\right)$ is determined from (12) by taking $\varepsilon=\varepsilon_{i}$. Obviously, each $n_{i}$ is picked out from the index set $\{1,2, \ldots, n-1\}$.

If necessary, we may take a subsequence $S_{J} \triangleq\left\{\varepsilon_{i_{j}}, j \geq 1\right\}$ such that both the coordinate index $n_{i}$ and $\hat{k}^{(i)}$ take constant values along $S_{J}$. In all of the following we base the analysis on the subsequence $S_{J}$; however, to simplify the notation we simply represent $S_{J}$ using the sequence $\left\{\varepsilon_{i}, i \geq 1\right\}$ and without loss of generality take $n_{i} \equiv 1$ and $\hat{k}^{(i)}=\hat{k}$.

Hence, we rewrite (14) as

$$
\hat{x}_{1}^{(i)}-\hat{y}_{1}^{(i)} \leq 0, \quad i \geq 1 .
$$


By definition, we have

$$
\Phi\left(\hat{x}^{(i)}, \hat{y}^{(i)}, \hat{k}\right)=\sup _{\bar{Q} \times \bar{Q} \times \Theta} \Phi(x, y, k) \geq \Phi\left(\hat{x}^{(i)}+\chi_{\delta}, \hat{y}^{(i)}, \hat{k}\right)
$$

for any $0<\delta \leq 1$, where we denote the vector $\chi_{\delta}=(\delta, 0, \ldots, 0)^{T}$. Since $\hat{x}^{(i)} \in \bar{Q}$, it is clear that $\hat{x}^{(i)}+\chi_{\delta} \in \bar{Q}$.

Step 2. Now we show that (15) together with (16) leads to a contradiction. By (16), we have

$$
\begin{aligned}
& \Phi\left(\hat{x}^{(i)}, \hat{y}^{(i)}, \hat{k}\right) \\
= & v_{1}\left(\hat{x}^{(i)}, \hat{k}\right)-v_{2}\left(\hat{y}^{(i)}, \hat{k}\right)-\left|\frac{\hat{x}^{(i)}-\hat{y}^{(i)}}{\varepsilon_{i}}-\tau \mathbf{1}_{n}\right|^{2}-\alpha\left[\zeta\left(\hat{x}^{(i)}\right)+\zeta\left(\hat{y}^{(i)}\right)\right] \\
\geq & v_{1}\left(\hat{x}^{(i)}+\chi_{\delta}, \hat{k}\right)-v_{2}\left(\hat{y}^{(i)}, \hat{k}\right)-\left|\frac{\hat{x}^{(i)}+\chi_{\delta}-\hat{y}^{(i)}}{\varepsilon_{i}}-\tau \mathbf{1}_{n}\right|^{2}-\alpha\left[\zeta\left(\hat{x}^{(i)}+\chi_{\delta}\right)+\zeta\left(\hat{y}^{(i)}\right)\right],
\end{aligned}
$$

which readily yields

$$
\begin{aligned}
T_{i} & \triangleq\left|\frac{\hat{x}_{1}^{(i)}-\hat{y}_{1}^{(i)}}{\varepsilon_{i}}-\tau\right|^{2}-\left|\frac{\hat{x}_{1}^{(i)}+\delta-\hat{y}_{1}^{(i)}}{\varepsilon_{i}}-\tau\right|^{2} \\
& \leq v_{1}\left(\hat{x}^{(i)}, \hat{k}\right)-v_{1}\left(\hat{x}^{(i)}+\chi_{\delta}, \hat{k}\right)+\alpha \zeta\left(\hat{x}^{(i)}+\chi_{\delta}\right)-\alpha \zeta\left(\hat{x}^{(i)}\right) .
\end{aligned}
$$

Obviously, for $\delta \in(0,1]$ we have $\left|\hat{x}^{(i)}\right| \vee\left|\hat{x}^{(i)}+\chi_{\delta}\right| \leq C_{\alpha}+1$ by Lemma 4 . Denote the constant $D_{\zeta, \alpha}=\sup _{|x| \leq C_{\alpha}+1}\left|\zeta^{\prime}(x)\right|$.

By (15) and then using the local Hölder and local Lipschitz continuity of $v_{1}$ and $\zeta$, respectively, it is easy to check that

$$
T_{i}=-\frac{\delta^{2}}{\varepsilon_{i}^{2}}+\frac{2 \delta}{\varepsilon_{i}}\left|\frac{\left|\hat{x}_{1}^{(i)}-\hat{y}_{1}^{(i)}\right|}{\varepsilon_{i}}+\tau\right| \leq \delta^{\gamma_{1}} \operatorname{Hol}\left(v_{1}, \gamma_{1}, C_{\alpha}+1\right)+\delta \alpha D_{\zeta, \alpha},
$$

where $\gamma_{1} \in(0,1]$ is the Hölder exponent for $v_{1}$, and therefore

$$
\frac{2 \delta \tau}{\varepsilon_{i}} \leq \frac{\delta^{2}}{\varepsilon_{i}^{2}}+\delta^{\gamma_{1}} \operatorname{Hol}\left(v_{1}, \gamma_{1}, C_{\alpha}+1\right)+\delta \alpha D_{\zeta, \alpha} .
$$

Since (18) holds for all $0<\delta \leq 1$, for the case with subscript index $i$, we take $\delta=\varepsilon_{i}^{\frac{2}{2-\gamma_{1}}}$ to obtain

$$
2 \tau \varepsilon_{i}^{\frac{-\gamma_{1}}{2-\gamma_{1}}} \leq 1+\operatorname{Hol}\left(v_{1}, \gamma_{1}, C_{\alpha}+1\right)+\varepsilon_{i}^{\frac{2\left(1-\gamma_{1}\right)}{2-\gamma_{1}}} \alpha D_{\xi, \alpha} .
$$

Letting $i \rightarrow \infty$, since $\tau>0$ is fixed, (19) leads to

$$
\operatorname{Hol}\left(v_{1}, \gamma_{1}, C_{\alpha}+1\right) \geq \infty,
$$

which is a contradiction since $v_{1} \in C_{p, H o l}^{l o c}(\bar{Q} \times \Theta)$ with the exponent $\gamma_{1}$.

Step 3. Combining Steps 1 and 2 above, we see that the initial assumption that (i) is invalid does not hold. Hence assertion (i) is proven. Since $\hat{y}_{i} \geq 0$ for $1 \leq i \leq n-1$, it follows that $\hat{x}_{i}>0$ for $i \leq n-1$, and consequently $\hat{x} \in Q$.

Notice that in order to derive the contradiction in Step 2 of the proof, it is necessary to take $\tau$ as an independent variable such that its magnitude may be controlled separately. 
4. Proof of Theorem 2. We give only the proof for Case (i) $\bar{Q}=\bar{Q}_{a}$, and Case (ii) $\bar{Q}=\bar{Q}_{b}$ can be treated without further difficulty. Let $(\hat{x}, \hat{y}, \hat{k})$ be obtained from (12). For given $\tau$ and $\alpha$, by Lemma 6 we can pick a sufficiently small $\varepsilon_{\tau, \alpha}$ depending on the pair $(\tau, \alpha)$ such that for all $0<\varepsilon \leq \varepsilon_{\tau, \alpha}$, its associated $(\hat{x}, \hat{y})$ is in the set $Q \times \bar{Q}$. In the following analysis we assume $\varepsilon \leq \varepsilon_{\tau, \alpha}$ is always satisfied. In particular, $\hat{x}$ is in the open set $Q$.

Let $\phi_{1}(x)=v_{2}(\hat{y})+\left|\frac{x-\hat{y}}{\varepsilon}-\tau \mathbf{1}_{n}\right|^{2}+\alpha[\zeta(x)+\zeta(\hat{y})]$ and $\phi_{2}(y)=v_{1}(\hat{x})-\mid \frac{\hat{x}-y}{\varepsilon}-$ $\left.\tau \mathbf{1}_{n}\right|^{2}-\alpha[\zeta(\hat{x})+\zeta(y)]$ be two test functions. Then on $\bar{Q}, v_{1}(x)-\phi_{1}(x)$ attains its maximum at $\hat{x} \in Q$, and $v_{2}(y)-\phi_{2}(y)$ attains its minimum at $\hat{y} \in \bar{Q}$. Hence we apply Definition 1 for viscosity sub/supersolutions to get

$$
\begin{aligned}
& \rho v_{1}(\hat{x}, \hat{k})-\inf _{u \in U_{\hat{k}}}\{[ {\left[2\left(\frac{\hat{x}-\hat{y}}{\varepsilon}-\tau \mathbf{1}_{n}\right)+\alpha \zeta^{\prime}(\hat{x})\right]^{T} F(\hat{x}, \hat{k}, u) } \\
&+\left.\Pi_{\theta}\left[v_{1}(\hat{x}, \cdot)\right](\hat{k})+L_{1}(\hat{x}, \hat{k}, u)\right\} \leq 0, \\
& \rho v_{2}(\hat{y}, \hat{k})-\inf _{u \in U_{\hat{k}}}\left\{\left[2\left(\frac{\hat{x}-\hat{y}}{\varepsilon}-\tau \mathbf{1}_{n}\right)+\alpha \zeta^{\prime}(\hat{y})\right]^{T} F(\hat{y}, \hat{k}, u)\right. \\
&\left.+\Pi_{\theta}\left[v_{2}(\hat{y}, \cdot)\right](\hat{k})+L_{2}(\hat{y}, \hat{k}, u)\right\} \geq 0 .
\end{aligned}
$$

The pair of inequalities (20) and (21) yields

$$
\begin{aligned}
& \rho v_{1}(\hat{x}, \hat{k})-\rho v_{2}(\hat{y}, \hat{k}) \\
\leq & \inf _{u \in U_{\hat{k}}}\left\{\left[2\left(\frac{\hat{x}-\hat{y}}{\varepsilon}-\tau \mathbf{1}_{n}\right)+\alpha \zeta^{\prime}(\hat{x})\right]^{T} F(\hat{x}, \hat{k}, u)\right. \\
& \left.+\Pi_{\theta}\left[v_{1}(\hat{x}, \cdot)\right](\hat{k})+L_{1}(\hat{x}, \hat{k}, u)\right\} \\
& \quad+\inf _{u \in U_{\hat{k}}}\left\{\left[2\left(\frac{\hat{x}-\hat{y}}{\varepsilon}-\tau \mathbf{1}_{n}\right)-\alpha \zeta^{\prime}(\hat{y})\right]^{T} F\left(v_{2}(\hat{y}, \cdot)\right](\hat{k})+L_{2}(\hat{y}, \hat{k}, u)\right\} \\
\leq & \sup _{u \in U_{\hat{k}}} 2\left|\frac{\hat{x}-\hat{y}}{\varepsilon}-\tau \mathbf{1}_{n}\right| \cdot|F(\hat{x}, \hat{k}, u)-F(\hat{y}, \hat{k}, u)| \\
& +\sup _{u \in U_{\hat{k}}} \alpha\left|F^{T}(\hat{x}, \hat{k}, u) \zeta^{\prime}(\hat{x})+F^{T}(\hat{y}, \hat{k}, u) \zeta^{\prime}(\hat{y})\right| \\
& +\sup _{u \in U_{\hat{k}}}\left[L_{1}(\hat{x}, \hat{k}, u)-L_{2}(\hat{y}, \hat{k}, u)\right]+\left\{\Pi_{\theta}\left[v_{1}(\hat{x}, \cdot)\right](\hat{k})-\Pi_{\theta}\left[v_{2}(\hat{y}, \cdot)\right](\hat{k})\right\} \\
\triangleq & A_{1}(\varepsilon, \hat{x}, \hat{y}, \hat{k})+A_{2}(\hat{x}, \hat{y}, \hat{k})+A_{3}(\hat{x}, \hat{y}, \hat{k})+A_{4}(\hat{x}, \hat{y}, \hat{k}) .
\end{aligned}
$$

Let $\alpha$ and $\tau$ be fixed first. Now in (22) we take a sequence $\varepsilon_{i} \downarrow 0$ with the associated $\left(\hat{x}_{i}, \hat{y}_{i}, \hat{k}_{i}\right)$ determined by (12). Here the subscript $i \geq 1$ in $\hat{x}_{i}$ is used to label the sequence and should not be confused as the index of a coordinate component. Since $\left|\hat{x}_{i}\right| \vee\left|\hat{y}_{i}\right| \leq C_{\alpha}$ for all $i \geq 1$ by Lemma 4 , there exists a subsequence denoted by 
$S_{x, y, k}=\left\{\left(\hat{x}_{i_{j}}, \hat{y}_{i_{j}}, \hat{k}_{i_{j}}\right), j \geq 1\right\}$, which converges to a limit $\left(x^{*}, x^{*}, k^{*}\right)$ in view of the fact that $\lim _{\varepsilon_{i} \rightarrow 0+}\left|\hat{x}_{i}-\hat{y}_{i}\right|=0$ by Lemma 5 . From (13) in the proof of Lemma 5 , it is seen that

$$
\left|\frac{\hat{x}-\hat{y}}{\varepsilon}-\tau \mathbf{1}_{n}\right| \leq \sqrt{\left(1+C_{\alpha}\right)\left[\operatorname{Hol}\left(v_{1}, \gamma_{1}, C_{\alpha}\right)+\operatorname{Hol}\left(v_{2}, \gamma_{2}, C_{\alpha}\right)\right]+n \tau^{2}},
$$

where the fixed parameter $\gamma_{i}$ is the Hölder exponent of $v_{i} \in C_{p, H o l}^{l o c}(\bar{Q} \times \Theta), i=1,2$. We combine (23) with the uniform continuity of $F(x, k, u)$ in $x$ for $|x| \leq C_{\alpha}$ (see (10)) to get

$$
\lim _{j \rightarrow \infty} A_{1}\left(\varepsilon_{i_{j}}, \hat{x}_{i_{j}}, \hat{y}_{i_{j}}, \hat{k}_{i_{j}}\right)=0 .
$$

Using the continuity of $F, L_{1}$, and $L_{2}$ with respect to $x$, it can be checked that both $A_{2}(\hat{x}, \hat{y}, \hat{k})$ and $A_{3}(\hat{x}, \hat{y}, \hat{k})$ are continuous in the arguments $(\hat{x}, \hat{y})$. Then we have

$$
\begin{aligned}
\lim _{j \rightarrow \infty}\left(A_{2}+A_{3}\right)\left(\hat{x}_{i_{j}}, \hat{y}_{i_{j}}, \hat{k}_{i_{j}}\right)= & 2 \alpha \sup _{u \in U_{k^{*}}}\left|\zeta^{\prime}\left(x^{*}\right) F\left(x^{*}, k^{*}, u\right)\right| \\
& +\sup _{u \in U_{k^{*}}}\left[L_{1}\left(x^{*}, k^{*}, u\right)-L_{2}\left(x^{*}, k^{*}, u\right)\right] .
\end{aligned}
$$

Now it readily follows from $(22)$ that

$$
\begin{aligned}
\rho v_{1}\left(x^{*}, k^{*}\right)-\rho v_{2}\left(x^{*}, k^{*}\right) \leq & 2 \alpha \sup _{u \in U_{k^{*}}}\left|\zeta^{\prime}\left(x^{*}\right) F\left(x^{*}, k^{*}, u\right)\right| \\
& +\sup _{u \in U_{k^{*}}}\left[L_{1}\left(x^{*}, k^{*}, u\right)-L_{2}\left(x^{*}, k^{*}, u\right)\right]+A_{4}\left(x^{*}, x^{*}, k^{*}\right) .
\end{aligned}
$$

On the other hand, for any $(x, x, k) \in \bar{Q} \times \bar{Q} \times \Theta$ and the set of parameters $\left(\varepsilon_{i_{j}}, \tau, \alpha\right)$, we have $\Phi(x, x, k) \leq \Phi\left(\hat{x}_{i_{j}}, \hat{y}_{i_{j}}, \hat{k}_{i_{j}}\right)$, i.e.,

$$
\begin{aligned}
& v_{1}(x, k)-v_{2}(x, k)-n \tau^{2}-2 \alpha \zeta(x) \\
\leq & v_{1}\left(\hat{x}_{i_{j}}, \hat{k}_{i_{j}}\right)-v_{2}\left(\hat{y}_{i_{j}}, \hat{k}_{i_{j}}\right)-\left|\frac{\hat{x}_{i_{j}}-\hat{y}_{i_{j}}}{\varepsilon_{i_{j}}}-\tau \mathbf{1}_{n}\right|^{2}-\alpha\left(\zeta\left(\hat{x}_{i_{j}}\right)+\zeta\left(\hat{y}_{i_{j}}\right)\right) \\
\leq & v_{1}\left(\hat{x}_{i_{j}}, \hat{k}_{i_{j}}\right)-v_{2}\left(\hat{y}_{i_{j}}, \hat{k}_{i_{j}}\right)-\alpha\left(\zeta\left(\hat{x}_{i_{j}}\right)+\zeta\left(\hat{y}_{i_{j}}\right)\right) .
\end{aligned}
$$

Taking $j \rightarrow \infty$ in (25) and invoking (24), we get

$$
\begin{aligned}
v_{1}(x, k)-v_{2}(x, k) \leq & v_{1}\left(x^{*}, k^{*}\right)-v_{2}\left(x^{*}, k^{*}\right)+n \tau^{2}+2 \alpha \zeta(x)-2 \alpha \zeta\left(x^{*}\right) \\
\leq & 2 \alpha \rho^{-1} \sup _{u \in U_{k^{*}}}\left|F\left(x^{*}, k^{*}, u\right)\right| \cdot\left|\zeta^{\prime}\left(x^{*}\right)\right| \\
& +\rho^{-1} \sup _{u \in U_{k^{*}}}\left[L_{1}\left(x^{*}, k^{*}, u\right)-L_{2}\left(x^{*}, k^{*}, u\right)\right] \\
& +\rho^{-1} A_{4}\left(x^{*}, x^{*}, k^{*}\right)+n \tau^{2}+2 \alpha \zeta(x)-2 \alpha \zeta\left(x^{*}\right) .
\end{aligned}
$$

By setting $x=x^{*}$ on both sides of (26), we have

$$
v_{1}\left(x^{*}, k\right)-v_{2}\left(x^{*}, k\right) \leq v_{1}\left(x^{*}, k^{*}\right)-v_{2}\left(x^{*}, k^{*}\right)+n \tau^{2}
$$


for all $k \in \Theta$, which gives

$$
\begin{aligned}
A_{4}\left(x^{*}, x^{*}, k^{*}\right) & =\sum_{k \neq k^{*}} \pi_{k^{*} k}\left[v_{1}\left(x^{*}, k\right)-v_{1}\left(x^{*}, k^{*}\right)\right]-\sum_{k \neq k^{*}} \pi_{k^{*} k}\left[v_{2}\left(x^{*}, k\right)-v_{2}\left(x^{*}, k^{*}\right)\right] \\
& =\sum_{k \neq k^{*}} \pi_{k^{*} k}\left\{\left[v_{1}\left(x^{*}, k\right)-v_{2}\left(x^{*}, k\right)\right]-\left[v_{1}\left(x^{*}, k^{*}\right)-v_{2}\left(x^{*}, k^{*}\right)\right]\right\} \\
& \leq n \tau^{2} \sum_{k \neq k^{*}} \pi_{k^{*} k}=n \tau^{2}\left|\pi_{k^{*} k^{*}}\right| .
\end{aligned}
$$

By use of the expression for $\zeta(x)$, it can be shown that

$$
\rho^{-1} \sup _{k \in \Theta} \sup _{u \in U_{k}}|F(x, k, u)| \cdot\left|\zeta^{\prime}(x)\right| \leq \zeta(x)
$$

for all $x \in \mathbb{R}$, and hence it follows from (27) that

$$
\begin{aligned}
v_{1}(x, k)-v_{2}(x, k) \leq & n \tau^{2}+2 \alpha \zeta(x)+\rho^{-1} \sup _{u \in U_{k^{*}}}\left[L_{1}\left(x^{*}, k^{*}, u\right)-L_{2}\left(x^{*}, k^{*}, u\right)\right] \\
& +\rho^{-1} n \tau^{2}\left|\pi_{k^{*} k^{*}}\right| \\
\leq & n \tau^{2}+2 \alpha \zeta(x)+\rho^{-1} \sup _{\bar{Q} \times \Theta u \in U_{k}}\left[L_{1}(x, k, u)-L_{2}(x, k, u)\right] \\
& +\rho^{-1} n \tau^{2} \max _{k}\left|\pi_{k k}\right| .
\end{aligned}
$$

Taking $\tau \rightarrow 0+$ and then $\alpha \rightarrow 0+$, we get

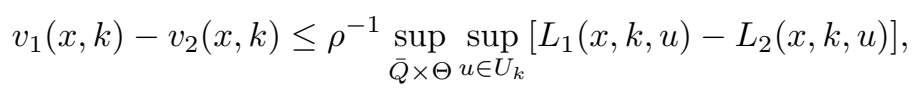

which completes the proof.

5. The value function as a constrained viscosity solution. In this section we give an existence result by showing that the value function $v$ associated with (1) and (2) gives a constrained viscosity solution. Under Definition 1, we first need to show that $v(x, k)$ is continuous in $x$, which is rather technical with the state constraints involved. To this end, we need some restrictions on the control set and the cost integrand in this general model. Here we take the state space to be $\bar{Q}_{b}$, and the case for $\bar{Q}_{a}$ can be treated analogously.

5.1. Hölder continuity of the value function and existence theorem. For deterministic systems, there has been a fair amount of work on continuity of infinite horizon value functions with state constraints, and usually only uniform continuity is proven; see [2] and references therein. By assuming a sufficiently large discount factor, Lipschitz continuity was obtained in [16, 12]. The proof in [12] made use of the viscosity sub/supersolution properties after showing that the value function is continuous and is the unique viscosity solution, and this method was extended to prove Hölder regularity in a state constrained diffusion model [13]. Here we take a different approach to obtain Hölder continuity by recursive upper bound estimates. Unlike $[12,16]$, our method does not involve the HJB equation and there is no restriction on the discount factor. that:

TheOrem 7. Suppose $\bar{Q}=\bar{Q}_{b}$, and $\left(\mathrm{A}^{\prime}\right)-(\mathrm{A} 2)$ hold. In addition, we assume

(i) each $U_{k}, k \in \Theta$, is equal to the same compact set $U \subset \mathbb{R}^{m}$; 
(ii) there exist positive constants $K_{i}<\infty, i=1,2,3$, such that

$$
\begin{gathered}
|F(x, u, k)-F(y, u, k)| \leq K_{1}|x-y|, \quad \forall x, y \in \mathbb{R}^{n}, u \in U, k \in \Theta, \\
\sup _{x \in \mathbb{R}^{n}, u \in U, k \in \Theta}|F(x, u, k)| \leq K_{2}, \quad \sup _{x \in \mathbb{R}^{n}, u \in U, k \in \Theta}|L(x, u, k)| \leq K_{3} ;
\end{gathered}
$$

(iii) there exist a continuous function $h: \partial \bar{Q} \rightarrow U$ and constant $\beta_{1}>0$ such that

$$
F_{i}(x, h(x), k) \geq \beta_{1}
$$

for $x \in \partial \bar{Q}, k \in \Theta$ and each $i \in\{1, \ldots, n\}$, where $\partial \bar{Q}$ denotes the boundary of $\bar{Q}$, and $F_{i}$ is the ith component of $F$.

Then for the value function $v$ defined in (2), we have the assertions:

(a) $v$ is bounded and Hölder continuous on $\bar{Q}$ (w.r.t. $x$ ), and

(b) $v$ is a unique constrained viscosity solution to (5) within the function class $C_{p, H o l}^{l o c}(\bar{Q} \times \Theta)$.

Remark. The proof of continuity relies on a modifying procedure, which consists of taking the control $u=h(\hat{x})$ for a short period when hitting $\hat{x} \in \partial \bar{Q}$ and switching back to a shifted version of the original control. Condition (i) ensures the admissibility of the modified control.

Remark. Condition (iii) is based on the idea of controllability on boundary initially due to Soner [22]; also see, e.g., [2, Chapter 5]. It means the state trajectory can be lifted inward at the boundary points and may be relaxed to other forms. For illustration, consider the example $\bar{Q}=[0, \infty) \times[0, \infty)$ and fix $r>0$. Then in addition to (28) being restricted on $x \in \partial \bar{Q} \cap\{x,|x| \leq r\}$, we may relax (iii) by only requiring $F_{1}(x, h(x), k) \geq \beta_{1}$ if $x=\left(0, x_{2}\right)$, where $x_{2} \geq r$, and a similar requirement for $F_{2}$ in the case $x=\left(x_{1}, 0\right), x_{1}>r$.

Remark. We establish uniqueness in the class $C_{p, H o l}^{l o c}(\bar{Q} \times \Theta)$, although $v$ is bounded.

For the value function $v$ and $r>0$, define

$$
\nu(r)=\sup \{|v(x, k)-v(y, k)|:|x-y|<r, \text { and } x, y \in \bar{Q}, k \in \Theta\} .
$$

Before proving Theorem 7, we give the following lemma on Hölder continuity. The proof is based on recursive estimation by gradually approaching the origin with small intervals for $r$. As an interesting byproduct for deterministic problems, Lemma 8 implies that the uniform continuity results in $[22,2]$ may be strengthened to Hölder regularity.

Lemma 8. For $\nu:(0, \infty) \rightarrow \mathbb{R}$ defined in (29), suppose $v$ is bounded and there exist constants $C>0,0<\alpha<1$, and $D>1$ such that

$$
\nu(r) \leq C r+\alpha \nu(D r)
$$

for all $r>0$, and let $\varepsilon_{0}$ be selected to satisfy either case (i) $0<\varepsilon_{0}<-(\ln \alpha) /(\ln D)$ and $\varepsilon_{0} \leq 1$ or case (ii) $\varepsilon_{0}=-(\ln \alpha) /(\ln D)$ provided that $-(\ln \alpha) /(\ln D)<1$. Then $v$ is Hölder continuous on $\bar{Q}$ with exponent $\varepsilon_{0}$, i.e.,

$$
\sup _{k \in \Theta, x \neq y} \frac{|v(x, k)-v(y, k)|}{|x-y|^{\varepsilon_{0}}}<\infty .
$$

Proof. Note that for sufficiently small $\varepsilon_{0}>0$, it always satisfies case (i). It is obvious that $\nu(r)$ monotonically increases with $r>0$ and is bounded since $v$ is 
bounded. For the estimate below, it suffices to restrict $r$ to the interval $(0,1]$. We denote $\Psi(r)=\nu(r) r^{-\varepsilon_{0}}$, and it follows from (30) that

$$
\Psi(r) \leq C r^{1-\varepsilon_{0}}+\alpha D^{\varepsilon_{0}} \Psi(D r) .
$$

We take the decomposition $(0,1]=\cup_{i=0}^{\infty} I_{i}$, where $I_{i}=\left(D^{-(i+1)}, D^{-i}\right]$. It is clear that $\sup _{r \in I_{0}} \Psi(r)<\infty$ since $v$ is bounded. For $r \in\left(D^{-(i+2)}, D^{-(i+1)}\right]$ and $i \geq 0$, we have $\operatorname{Dr} \in\left(D^{-(i+1)}, D^{-i}\right]$, which in conjunction with (31) gives

$$
\sup _{r \in I_{i}} \Psi(r) \leq C D^{-\left(1-\varepsilon_{0}\right) i}+\alpha D^{\varepsilon_{0}} \sup _{r \in I_{i-1}} \Psi(r), \quad i \geq 1 .
$$

By iterating (32), it follows that

$$
\sup _{r \in I_{k}} \Psi(r) \leq C \sum_{i=0}^{k-1} D^{-\left(1-\varepsilon_{0}\right)(k-i)}\left(\alpha D^{\varepsilon_{0}}\right)^{i}+\left(\alpha D^{\varepsilon_{0}}\right)^{k} \sup _{r \in I_{0}} \Psi(r) .
$$

Denote $S_{k}=C \sum_{i=0}^{k-1} D^{-\left(1-\varepsilon_{0}\right)(k-i)}\left(\alpha D^{\varepsilon_{0}}\right)^{i}$. For case (i), we have $\alpha D^{\varepsilon_{0}}<1$ and $S_{k} \leq C \sum_{i=0}^{\infty}\left(\alpha D^{\varepsilon_{0}}\right)^{i}=C\left(1-\alpha D^{\varepsilon_{0}}\right)^{-1}$. For case (ii), we have $\alpha D^{\varepsilon_{0}}=1,1-\varepsilon_{0}>0$, and therefore $S_{k}=C \sum_{i=0}^{k-1} D^{-\left(1-\varepsilon_{0}\right)(k-i)}<C\left(D^{1-\varepsilon_{0}}-1\right)^{-1}$.

Combining cases (i) and (ii), we see that the right-hand side of (33) is bounded by a constant independent of $k$. Hence we conclude that

$$
\sup _{k \in \Theta, 0<|x-y| \leq 1} \frac{|v(x, k)-v(y, k)|}{|x-y|^{\varepsilon_{0}}} \leq \sup _{r \in(0,1]} \Psi(r)<\infty,
$$

for $\varepsilon_{0}$ determined by either case (i) or case (ii), which implies the Hölder continuity of $v$.

Remark. If $\alpha D<1$ holds, (30) implies Lipschitz continuity of $v$ since we may take $\varepsilon_{0}=1$ for case (i).

5.2. Proof of Theorem 7. We begin by proving assertion (a), which is broken into two steps.

Step 1. Let $(z, k) \in \bar{Q} \times \Theta$ be the initial condition at $t=0$ and $\tau_{k}$ the first jump time of $\theta(t)$ starting from $k \in \Theta$. If $k$ is an absorbing state of $\theta(t)$, we simply have $\tau_{k} \equiv \infty$. We write $\mathcal{U}_{z, k}$ as $\mathcal{U}_{z}$ since all $U_{k}=U$. Following the same method as in $[22,2]$, we first show that there exist a small $t^{*}>0$ and a constant $C_{1}>0$ such that for all $(z, k) \in \bar{Q} \times \Theta$ and $u$ adapted to $\mathcal{F}_{t}=\sigma(\theta(s), s \leq t)$, there is $\bar{u} \in \mathcal{U}_{z}$ such that

$$
\left|J_{t^{*} \wedge \tau_{k}}(z, k, u)-J_{t^{*} \wedge \tau_{k}}(z, k, \bar{u})\right| \leq C_{1} \sup _{0 \leq t \leq t^{*} \wedge \tau_{k}} d(X(t, z, k, u), \bar{Q}),
$$

where $J_{t^{*} \wedge \tau_{k}}=\int_{0}^{t^{*} \wedge \tau_{k}} e^{-\rho t} L(X, u, \theta)(t) d t$ with the initial condition $(z, k)$ at $t=0$, $X(t, z, k, u)$ is the state at time $t$ associated with the initial condition $(z, k)$ and control $u$, and $d(X(t, z, k, u), \bar{Q})$ denotes the distance between the state and $\bar{Q}$ on that particular sample $\omega$.

For proving (34), we need to determine two constants $t^{*}, \kappa>0$ below. Before proceeding to do so, we set $t_{0}=\tau_{z, k, u} \wedge t^{*}$, where we define $\tau_{z, k, u}=\inf \{0 \leq$ $\left.t \leq t^{*}, X(t, z, k, u) \in \partial \bar{Q}\right\}$, if $X(t, z, k, u)$ reaches $\partial \bar{Q}$ before $t^{*}$, or $\tau_{z, k, u}=t^{*}$, if $X(t, z, k, u) \in Q$ for all $t \leq t^{*}$, and $\varepsilon=\sup _{0 \leq t \leq t^{*} \wedge \tau_{k}} d(X(t, z, k, u), \bar{Q})$. Let $u$ be any control adapted to $\mathcal{F}_{t}$. We construct the new control

$$
\hat{u}(t)=u(t) 1_{\left[0, t_{0}\right)}+h\left(X\left(t_{0}\right)\right) 1_{\left[t_{0}, t_{0}+\kappa \varepsilon\right]}+u(t-k \varepsilon) 1_{\left(t_{0}+\kappa \varepsilon, \infty\right)},
$$


which is adapted to $\mathcal{F}_{t}$. Below we will show that $X(t, z, k, \hat{u}) \in \bar{Q}$ for all $t \leq t^{*} \wedge \tau_{k}$ after $t^{*}$ and $\kappa$ are appropriately chosen; by repeating this construction procedure on successive small intervals covering $[0, \infty)$, we obtain $\bar{u} \in \mathcal{U}_{z}$ and $\bar{u} \equiv \hat{u}$ on $\left[0, t^{*} \wedge \tau_{k}\right]$. Once this is done, the nonemptiness of $\mathcal{U}_{z}$ and $\sup _{\bar{Q} \times U \times \Theta}|L(x, u, k)|<\infty$ implies that $v$ is bounded on $\bar{Q} \times \Theta$.

By uniform continuity of $F$ (w.r.t. $x$ ), there exists $\delta>0$ such that $F_{i}\left(z, h\left(z_{0}\right), k\right) \geq$ $\beta_{1} / 2$ provided that $\left|z-z_{0}\right| \leq \delta$ and $z_{0} \in \partial \bar{Q}$.

We first make the restriction $t^{*}<\delta /\left(2 K_{2}\right)$. If $d(z, \partial \bar{Q}) \geq \delta / 2$, then $t_{0}=t^{*}$ and $X(t, z, k, u) \in Q$ for $t \leq t^{*}$. Now it suffices to consider the case $t_{0}=\tau_{z, k, u}<$ $t^{*}<\delta /\left(2 K_{2}\right)$. It can be checked that $\left|X(t, z, k, \hat{u})-X\left(t_{0}, z, k, u\right)\right| \leq \delta$, and therefore $F_{i}\left(X(t, z, k, \hat{u}), h\left(X\left(t_{0}, z, k, u\right)\right), \theta_{t}\right) \geq \beta_{1} / 2,1 \leq i \leq n$, for $t \leq t^{*}$. It is obvious that $X(t, z, k, \hat{u}) \in \bar{Q}$ for all $t \leq t^{*} \wedge\left(t_{0}+\kappa \varepsilon\right)$ by the construction of $\hat{u}$; for the case $t^{*} \wedge \tau_{k} \leq\left(t_{0}+\kappa \varepsilon\right)$, we immediately have $X(t, z, k, \hat{u}) \in \bar{Q}$ for $t \leq t^{*} \wedge \tau_{k}$.

If $t^{*} \wedge \tau_{k}>\left(t_{0}+\kappa \varepsilon\right)$, we apply a similar method as in [2, pp. 272-274] to show that $X(t, z, k, \hat{u}) \in \bar{Q}$ for all $t \leq t^{*} \wedge \tau_{k}$. Indeed, for $t_{0}+\kappa \varepsilon \leq t \leq t^{*} \wedge \tau_{k}$, we may write

$$
\begin{aligned}
\hat{X}_{t}^{(i)} \geq & X_{t_{0}}^{(i)}+\frac{\beta_{1}}{2} \kappa \varepsilon+\int_{t_{0}+\kappa \varepsilon}^{t} F_{i}\left(\hat{X}_{s}, \hat{u}_{s}, \theta_{s}\right) d s \\
= & X_{t_{0}}^{(i)}+\frac{\beta_{1}}{2} \kappa \varepsilon+\int_{t_{0}+\kappa \varepsilon}^{t} F_{i}\left(X_{s-\kappa \varepsilon}, \hat{u}_{s}, \theta_{s}\right) d s \\
& +\int_{t_{0}+\kappa \varepsilon}^{t} F_{i}\left(\hat{X}_{s}, \hat{u}_{s}, \theta_{s}\right) d s-\int_{t_{0}+\kappa \varepsilon}^{t} F_{i}\left(X_{s-\kappa \varepsilon}, \hat{u}_{s}, \theta_{s}\right) d s,
\end{aligned}
$$

where $\hat{X}_{t}=X(t, z, k, \hat{u}), X_{t}=X(t, z, k, u)$, and we use the superscript $i$ in $\hat{X}_{t}$, $X_{t}$ to denote the $i$ th component in the vector. Recalling the construction of $\hat{u}$ for $t_{0}+\kappa \varepsilon \leq t \leq t^{*} \wedge \tau_{k}$, we have

$$
\begin{aligned}
X_{t_{0}}^{(i)}+\int_{t_{0}+\kappa \varepsilon}^{t} F_{i}\left(X_{s-\kappa \varepsilon}, \hat{u}_{s}, \theta_{s}\right) d s & =X_{t_{0}}^{(i)}+\int_{t_{0}}^{t-\kappa \varepsilon} F_{i}\left(X_{s}, u_{s}, \theta_{s+\kappa \varepsilon}\right) d s \\
& =X_{t_{0}}^{(i)}+\int_{t_{0}}^{t-\kappa \varepsilon} F_{i}\left(X_{s}, u_{s}, \theta_{s}\right) d s \\
& =X_{t-\kappa \varepsilon}^{(i)} \geq-\varepsilon,
\end{aligned}
$$

where the inequality in (37) holds by the definition of $\varepsilon$. On the other hand, by the Lipschitz continuity of $F_{i}$, we have

$$
\begin{aligned}
& \left|\int_{t_{0}+\kappa \varepsilon}^{t} F_{i}\left(\hat{X}_{s}, \hat{u}_{s}, \theta_{s}\right) d s-\int_{t_{0}+\kappa \varepsilon}^{t} F_{i}\left(X_{s-\kappa \varepsilon}, \hat{u}_{s}, \theta_{s}\right) d s\right| \\
\leq & K_{1} \int_{t_{0}+\kappa \varepsilon}^{t}\left|\hat{X}_{s}-X_{s-\kappa \varepsilon}\right| d s \\
\leq & K_{1}\left|\hat{X}_{t_{0}+\kappa \varepsilon}-X_{t_{0}}\right| \int_{t_{0}+\kappa \varepsilon}^{t} e^{K_{1}\left(s-t_{0}-\kappa \varepsilon\right)} d s \\
\leq & \left|\hat{X}_{t_{0}+\kappa \varepsilon}-X_{t_{0}}\right|\left(e^{K_{1}\left(t-t_{0}-\kappa \varepsilon\right)}-1\right) \leq \kappa \varepsilon K_{2}\left(e^{K_{1}\left(t-t_{0}-\kappa \varepsilon\right)}-1\right),
\end{aligned}
$$

where (38) is obtained by estimating $\left|\hat{X}_{s}-X_{s-\kappa \varepsilon}\right|$ via Gronwall inequality.

Hence for $t_{0}+\kappa \varepsilon \leq t \leq t^{*} \wedge \tau_{k}$, it follows from (36), (37), and (39) that

$$
\hat{X}_{t}^{(i)} \geq \frac{\beta_{1}}{2} \kappa \varepsilon-\varepsilon-\kappa \varepsilon K_{2}\left(e^{K_{1}\left(t-t_{0}-\kappa \varepsilon\right)}-1\right) \text {. }
$$


We conclude that if we take $t^{*}=\min \left\{\frac{1}{K_{1}} \ln \left(\frac{\beta_{1}}{4 K_{2}}+1\right), \frac{\delta}{3 K_{2}}\right\}$ and $\kappa=4 / \beta_{1}$, then $\hat{X}_{t}=X(t, z, k, \hat{u}) \in \bar{Q}$ for all $t \leq t^{*} \wedge \tau_{k}$. This completes the construction of $\hat{u}$ and subsequently that of $\bar{u} \in \mathcal{U}_{z}$. The inequality (34) is obtained by use of the boundedness of $L$ and simple integral estimates as in [2].

Step 2. Now we proceed to prove continuity of the value function. Let $t^{*}$ be determined as above and $|z-y|<r$, where $z, y \in \bar{Q}$. For any $\delta_{1}>0$, by the optimality principle we may find $u \in \mathcal{U}_{z}$ such that

$$
E\left[J_{t^{*} \wedge \tau_{k}}(z, k, u)+e^{-\rho\left(t^{*} \wedge \tau_{k}\right)} v\left(X\left(t^{*} \wedge \tau_{k}, z, k, u\right), \theta_{t^{*} \wedge \tau_{k}}\right)\right] \leq v(z, k)+\delta_{1} .
$$

Based on $u$ we construct $\bar{u} \in \mathcal{U}_{y}$ by use of (35). By basic estimates similar to those in [2, pp. 274-275] we can show $\left|X\left(t^{*} \wedge \tau_{k}, z, k, u\right)-X\left(t^{*} \wedge \tau_{k}, y, k, \bar{u}\right)\right| \leq C_{2} r$ and $\left|J_{t^{*} \wedge \tau_{k}}(z, k, u)-J_{t^{*} \wedge \tau_{k}}(y, k, \bar{u})\right| \leq C_{3} r$ for constants $C_{2}>1, C_{3}>0$. Subsequently, we get

$$
\begin{aligned}
v(y, k)-v(z, k) \leq & E\left[J_{t^{*} \wedge \tau_{k}}(y, k, \bar{u})+e^{-\rho\left(t^{*} \wedge \tau_{k}\right)} v\left(X\left(t^{*} \wedge \tau_{k}, y, k, \bar{u}\right), \theta_{t^{*} \wedge \tau_{k}}\right)\right] \\
& -E\left[J_{t^{*} \wedge \tau_{k}}(z, k, u)+e^{-\rho\left(t^{*} \wedge \tau_{k}\right)} v\left(X\left(t^{*} \wedge \tau_{k}, z, k, u\right), \theta_{t^{*} \wedge \tau_{k}}\right)\right]+\delta_{1} .
\end{aligned}
$$

By arbitrariness of $\delta_{1}>0$, it follows that $\nu(r) \leq C_{3} r+E e^{-\rho\left(t^{*} \wedge \tau_{k}\right)} \nu\left(C_{2} r\right)$. If $k$ is an absorbing state, we have $0<E e^{-\rho\left(t^{*} \wedge \tau_{k}\right)}=e^{-\rho t^{*}} \triangleq \alpha_{1}<1$; otherwise, $\tau_{k}$ is exponentially distributed with the density function $\lambda_{k} e^{-\lambda_{k} t}$ on $[0, \infty)$, where $\lambda_{k}=$ $-\pi_{k k}>0$, and we have $E e^{-\rho\left(t^{*} \wedge \tau_{k}\right)}=\lambda_{k} /\left(\lambda_{k}+\rho\right)+\rho e^{-\left(\lambda_{k}+\rho\right) t^{*}} /\left(\lambda_{k}+\rho\right) \leq 1-\rho(1-$ $\left.e^{-\rho t^{*}}\right) /\left(\lambda^{*}+\rho\right) \triangleq \alpha_{2}<1$, where $\lambda^{*}=\max _{k \in \Theta}\left\{\left|\pi_{k k}\right|\right\}$. Hence we obtain

$$
\nu(r) \leq C_{3} r+\alpha \nu\left(C_{2} r\right),
$$

where $\alpha=\max \left\{\alpha_{1}, \alpha_{2}\right\}<1$ and $C_{2}>1$. This leads to Hölder continuity of $v$ by Lemma 8.

For proving assertion (b), the verification of the constrained viscosity solution property is similar to the state unconstrained case, and we omit the details here. Uniqueness of the constrained viscosity solution follows from Theorem 3.

Remark. For brevity, we only give a detailed proof of existence in Theorem 11 which deals with a composite mode variable, and the steps there can be adapted to this theorem in a straightforward manner to verify the constrained viscosity solution property of the value function.

Remark. For the estimation in section 5.2, it is necessary to apply truncation by the jump time $\tau_{k}$; otherwise the derivation for (37) and (38) is invalid. Also note that $F$ in the dynamics and the cost integrand $L$ are restricted to be bounded. With a more general growth condition in $x$ for $F$ and $L$, the corresponding ODE estimates will be more challenging.

6. An optimal scheduling problem. As an application of the results in section 3, we consider a fluid buffer control problem for data traffic relay arising in communication networks; relevant background information can be found in the wireless application work [10] and references therein. Suppose a relay buffer is deployed to connect a source and a destination; see Figure 2. The incoming and outgoing links are described by two continuous time independent finite state Markov chains $y(t)$ and $z(t)$, indicating a certain channel quality. Suppose that $y(t)$ and $z(t)$ have state spaces $S_{y}=\left\{1, \ldots, m_{1}\right\}, S_{z}=\left\{1, \ldots, m_{2}\right\}$ and transition probability rate matrices $\Pi_{y}=\left(p_{i j}\right)_{m_{1} \times m_{1}}, \Pi_{z}=\left(q_{i j}\right)_{m_{2} \times m_{2}}$, respectively. 


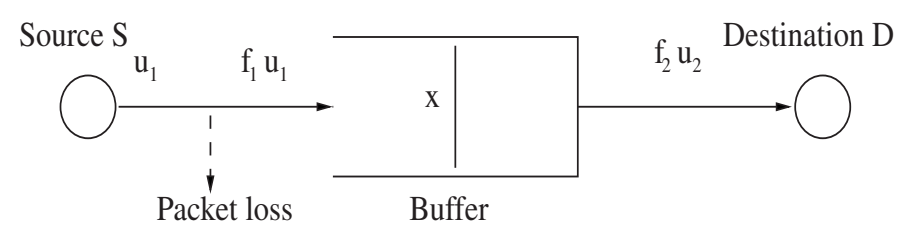

FIG. 2. The fluid buffer model.

Let $X \geq 0$ denote the buffer level (number of data packets), and let $u_{i}, i=1,2$, be the transmission rate (packets per second) at the incoming and outgoing links, respectively. Write the buffer level dynamics in the form:

$$
\frac{d X(t)}{d t}=\left[u_{1} f_{1}\left(y, u_{1}\right)-u_{2} f_{2}\left(z, u_{2}\right)\right](t) \triangleq F(y, z, u)(t), \quad t \geq 0,
$$

subject to $X \geq 0$. Here $f_{i}, i=1,2$, is the success probability of transmission given the link state $y$ or $z$ and rate $u_{i}$, and $u=\left[u_{1}, u_{2}\right]^{T}$. Notice that the buffer level decrease rate is only a fraction of $u_{2}$ since a packet which fails to reach the destination is not immediately deleted and will stay for retransmission. Furthermore, for limiting interference, at a given time it is allowed to transmit at only one link, either the incoming or the outgoing link [10].

We define the discounted utility function as

$$
\begin{aligned}
& J_{u t}(x, i, j, u)= \\
& E\left[\int_{0}^{\infty} e^{-\rho t}\left[F_{1}\left(y, u_{1}\right)+F_{2}\left(z, u_{2}\right)-\lambda X\right](t) d t \mid X(0)=x, y(0)=i, z(0)=j\right],
\end{aligned}
$$

where $\rho>0, \lambda>0, F_{1}\left(y, u_{1}\right) \triangleq u_{1} f_{1}\left(y, u_{1}\right)$, and $F_{2}\left(z, u_{1}\right) \triangleq u_{2} f_{2}\left(z, u_{2}\right)$. The term $\lambda X$ corresponds to a linear holding cost for the buffer level. The function $J^{0}\left(y, u_{1}, z, u_{2}\right)=F_{1}+F_{2}$ is naturally interpreted as the instantaneous aggregate utility of the buffer in successfully transporting infinitesimal traffic volume by one hop - operating in either the receiving or the transmitting mode.

Let $U_{i}=\left[\underline{R}_{i}, \bar{R}_{i}\right], i=1,2$, where $0<\underline{R}_{i}<\bar{R}_{i}<\infty$. The control at a given time is denoted as $u=\left(u_{1}, u_{2}\right)^{T} \in U \triangleq\left(U_{1} \times\{0\}\right) \cup\left(\{0\} \times U_{2}\right)$. Define the $\sigma$-algebra $\mathcal{F}_{t}=\sigma(y(s), z(s), s \leq t)$.

The objective for the optimal scheduling problem is to maximize $J_{u t}$ or, equivalently, to minimize $-J_{u t}$. Specializing the general formulation in section 2 to the current setting, we denote the admissible control set $\mathcal{U}_{x, i, j}$ with the initial condition $(x, i, j)$ for $(X(t), y(t), z(t))$. Let $v(x, i, j)$ denote the value function for minimizing $J(x, i, j, u) \triangleq-J_{u t}(x, i, j, u)$, where $x \in[0, \infty), i \in S_{y}$, and $j \in S_{z}$, and write $L(x, i, j, u)=-F_{1}\left(i, u_{1}\right)-F_{2}\left(j, u_{2}\right)+\lambda x$. The following assumption is used throughout this section.

(A3) $f_{1}\left(\right.$ resp., $\left.f_{2}\right)$ is a function mapping $S_{y} \times U_{1}$ (resp., $\left.S_{z} \times U_{2}\right) \rightarrow[0,1]$ and is continuous in $u_{1}$ (resp., $u_{2}$ ).

6.1. Existence and uniqueness of viscosity solutions. For a function $\varphi(x, i, j)$ continuous in $x \in[0, \infty)$, define the operator

$$
\begin{aligned}
{[\Pi \varphi(x, \cdot, \cdot)](i, j)=} & \sum_{i^{\prime} \neq i} p_{i i^{\prime}}\left[\varphi\left(x, i^{\prime}, j\right)-\varphi(x, i, j)\right] \\
& +\sum_{j \neq j^{\prime}} q_{j j^{\prime}}\left[\varphi\left(x, i, j^{\prime}\right)-\varphi(x, i, j)\right],
\end{aligned}
$$


where $\Pi_{y}=\left(p_{i j}\right)_{m_{1} \times m_{1}}$ and $\Pi_{z}=\left(q_{i j}\right)_{m_{2} \times m_{2}}$. For the value function $v$, we write the HJB equation in the compact form:

$$
\begin{aligned}
\rho v(x, i, j) & =[\Pi v(x, \cdot, \cdot)](i, j)+\inf _{u \in U}\left[v_{x}(x, i, j)\left(F_{1}\left(i, u_{1}\right)-F_{2}\left(j, u_{2}\right)\right)+L(x, i, j, u)\right] \\
(44) & =H\left(x, i, j, v_{x}(x, i, j), v(x, \cdot, \cdot)\right) .
\end{aligned}
$$

Notice that after introducing a new set of indices for the joint Markov chain $(y, z)$ with its associated transition probability rate matrix, (44) can be written in the standard form in section 2. The details for such a conversion are omitted here. Before proving that the value function $v$ is a constrained viscosity solution to (44), we show that $v$ is continuous in $x$.

Lemma 9. Let $0 \leq \hat{x}<x<\infty$ be given and $(y(0), z(0))=(i, j) \in S_{y} \times S_{z}$ be fixed. For any $u \in \mathcal{U}_{x, i, j}$, there exists $\hat{u} \in \mathcal{U}_{\hat{x}, i, j}$ such that

(i) $\sup _{t \geq 0}|\hat{X}(t)-X(t)| \leq|\hat{x}-x|$, and

(ii) with probability one, we have

$$
\left|\int_{0}^{t}\left\{\left[F_{1}\left(y, \hat{u}_{1}\right)-F_{1}\left(y, u_{1}\right)\right]+\left[F_{2}\left(z, u_{2}\right)-F_{2}\left(z, \hat{u}_{2}\right)\right]\right\}(s) d s\right| \leq 2|\hat{x}-x|
$$

for all $t>0$, where $X(t)$ and $\hat{X}(t)$ are, respectively, the solution associated with the control $u, \hat{u}$ and the initial condition $x, \hat{x}$.

Proof. For $u \in \mathcal{U}_{x, i, j}$, let $X(t, \hat{x}, u)$ denote the state at time $t$ with the initial condition $\hat{x} \geq 0$ and control $u$. Let $\tau_{1}=\inf \{t \geq 0 \mid X(t, \hat{x}, u)=0\}$ and $\tau_{1}=\infty$ on $\{X(t, \hat{x}, u)>0, \forall t \geq 0\}$. Denote $\delta=|x-\hat{x}| /\left(\bar{R}_{1}+\bar{R}_{2}\right)$. We construct the control $u^{(1)}$ as follows:

$$
u^{(1)}(t)= \begin{cases}u(t) & \text { for } t<\tau_{1} \\ {\left[\bar{R}_{1}, 0\right]^{T}} & \text { for } t \in\left[\tau_{1}, \tau_{1}+\delta\right), \\ u(t) & \text { for } t \geq \tau_{1}+\delta\end{cases}
$$

Suppose $\tau_{k}$ and $u^{(k)}, k \geq 1$, have been constructed. Define $\tau_{k+1}=\inf \left\{t \geq \tau_{k}+\right.$ $\left.\delta \mid X\left(t, \hat{x}, u^{(k)}\right)=0\right\}$ on $\left\{\tau_{k}<\infty\right\}, \tau_{k+1}=\infty$ on $\left\{\tau_{k}=\infty\right\} \cup\left\{\tau_{k}<\infty\right.$, and $\left.X\left(t, \hat{x}, u^{(k)}\right)>0, \forall t \geq \tau_{k}+\delta\right\}$; define $u^{(k+1)}$ by setting $\left(u, \tau_{1}\right)$ as $\left(u^{(k)}, \tau_{k+1}\right)$ on the right-hand side of (45). This procedure may be terminated if the stopping time $\tau_{k}$ at a certain stage $k$ equals $\infty$ with probability one. Let $\hat{u}(t)=u^{(k)}(t)$ for $t \leq \tau_{k+1}$, and it can be shown that this gives a well-defined control on $[0, \infty)$ and $\hat{u} \in \mathcal{U}_{\hat{x}, i, j}$.

In (46) below, $X(t)$ and $\hat{X}(t)$ are associated with $u$ and $\hat{u}$, respectively. By the construction of $\hat{u}$, it is easy to check that $\hat{X}(t)-X(t) \geq-|\hat{x}-x|$ for all $t \geq 0$. Now we show that for all $t \geq 0, \hat{X}(t)-X(t) \leq|\hat{x}-x|$, which obviously holds for $t \leq \tau_{1}$. Suppose $t \in\left[\tau_{k}, \tau_{k+1}\right)$. Since $0=\hat{X}\left(\tau_{k}\right) \leq X\left(\tau_{k}\right)$, we have

$$
\begin{aligned}
\hat{X}(t)-X(t) & =\hat{X}\left(\tau_{k}\right)+\int_{\tau_{k}}^{t}\left(\hat{F}_{1}-\hat{F}_{2}\right)(s) d s-X\left(\tau_{k}\right)-\int_{\tau_{k}}^{t}\left(F_{1}-F_{2}\right)(s) d s \\
& \leq \int_{\tau_{k}}^{t \wedge\left(\tau_{k}+\delta\right)}\left(\hat{F}_{1}-\hat{F}_{2}\right)(s) d s-\int_{\tau_{k}}^{t \wedge\left(\tau_{k}+\delta\right)}\left(F_{1}-F_{2}\right)(s) d s \\
& =\int_{\tau_{k}}^{t \wedge\left(\tau_{k}+\delta\right)}\left(\hat{F}_{1}-F_{1}\right)(s) d s+\int_{\tau_{k}}^{t \wedge\left(\tau_{k}+\delta\right)}\left(F_{2}-\hat{F}_{2}\right)(s) d s \\
& \leq \bar{R}_{1} \delta+\bar{R}_{2} \delta=|\hat{x}-x|,
\end{aligned}
$$


where we denote $F_{1}=F_{1}\left(y, u_{1}\right), \hat{F}_{1}=F_{1}\left(y, \hat{u}_{1}\right), F_{2}=F_{2}\left(z, u_{2}\right)$, etc. Hence $\sup _{t \geq 0}|\hat{X}(t)-X(t)| \leq|\hat{x}-x|$, and (i) follows. On the other hand, we have

$$
\hat{X}(t)-X(t)=\hat{x}-x+\int_{0}^{t}\left[\left(\hat{F}_{1}-F_{1}\right)+\left(F_{2}-\hat{F}_{2}\right)\right](s) d s .
$$

By use of (47) and (i) we get

$$
\sup _{t \geq 0}\left|\int_{0}^{t}\left[\left(\hat{F}_{1}-F_{1}\right)+\left(F_{2}-\hat{F}_{2}\right)\right](s) d s\right| \leq \sup _{t \geq 0}|\hat{X}(t)-X(t)|+|\hat{x}-x| \leq 2|\hat{x}-x|,
$$

and (ii) follows.

LEMma 10. The value function $v(x, i, j)$ is Lipschitz continuous with respect to $x \in[0, \infty)$.

Proof. Take $0 \leq \hat{x}<x$. We need to estimate $|v(\hat{x}, i, j)-v(x, i, j)|$. For any $\varepsilon>0$, there exists $u_{\varepsilon} \in \overline{\mathcal{U}}_{x, i, j}$ such that $v(x, i, j) \leq J\left(x, i, j, u_{\varepsilon}\right) \leq v(x, i, j)+\varepsilon$. Based on $u_{\varepsilon}$, we construct $\hat{u}_{\varepsilon} \in \mathcal{U}_{\hat{x}, i, j}$ satisfying (i) and (ii) in Lemma 9. Using the same set of notation as in (46) and noticing $\hat{F}_{1}-F_{1} \geq 0, F_{2}-\hat{F}_{2} \geq 0$, we have

$$
\begin{aligned}
& \left|\int_{0}^{\infty} e^{-\rho t}\left(\hat{F}_{1}+\hat{F}_{2}\right) d t-\int_{0}^{\infty} e^{-\rho t}\left(F_{1}+F_{2}\right) d t\right| \\
= & \left|\int_{0}^{\infty} e^{-\rho t}\left(\hat{F}_{1}-F_{1}\right) d t+\int_{0}^{\infty} e^{-\rho t}\left(\hat{F}_{2}-F_{2}\right) d t\right| \\
\leq & \left|\int_{0}^{\infty} e^{-\rho t}\left(\hat{F}_{1}-F_{1}\right) d t+\int_{0}^{\infty} e^{-\rho t}\left(F_{2}-\hat{F}_{2}\right) d t\right| \\
\leq & \left|\int_{0}^{\infty}\left[\left(\hat{F}_{1}-F_{1}\right)+\left(F_{2}-\hat{F}_{2}\right)\right] d t\right| \leq 2|\hat{x}-x|,
\end{aligned}
$$

where the last inequality follows from Lemma 9 (ii).

By (48) and Lemma 9(i), we can check that

$$
\begin{aligned}
\left|J\left(\hat{x}, i, j, \hat{u}_{\varepsilon}\right)-J\left(x, i, j, u_{\varepsilon}\right)\right| & \leq 2|\hat{x}-x|+E \int_{0}^{\infty} e^{-\rho t} \lambda|\hat{X}(t)-X(t)| d t \\
& \leq(2+\lambda / \rho)|\hat{x}-x| .
\end{aligned}
$$

Hence $v(\hat{x}, i, j) \leq v(x, i, j)+\varepsilon+(2+\lambda / \rho)|\hat{x}-x|$. On the other hand, suppose $\hat{u}_{\varepsilon}$ has been found such that $J\left(\hat{x}, i, j, \hat{u}_{\varepsilon}\right) \leq v(\hat{x}, i, j)+\varepsilon$; then obviously $\hat{u}_{\varepsilon} \in \mathcal{U}_{x, i, j}$, and we can verify that $J\left(x, i, j, \hat{u}_{\varepsilon}\right) \leq J\left(\hat{x}, i, j, \hat{u}_{\varepsilon}\right)+(\lambda / \rho)|\hat{x}-x|$ and hence $v(x, i, j) \leq$ $v(\hat{x}, i, j)+\varepsilon+(\lambda / \rho)|\hat{x}-x|$.

Thus $|v(\hat{x}, i, j)-v(x, i, j)| \leq(2+\lambda / \rho)|\hat{x}-x|+\varepsilon$. Since $\varepsilon>0$ is arbitrary, we get $|v(\hat{x}, i, j)-v(x, i, j)| \leq(2+\lambda / \rho)|\hat{x}-x|$, and the lemma follows.

TheOREM 11. The value function $v:[0, \infty) \times S_{y} \times S_{z} \rightarrow \mathbb{R}$ is a unique constrained viscosity solution to (44) in the function class $C_{p, L i p}^{\text {loc }}\left([0, \infty) \times S_{y} \times S_{z}\right)$.

Proof. See the appendix.

7. Application to stochastic manufacturing systems. In this section we consider production rate control involving $n$ machines in a tandem queue with $n-1$ buffers between neighboring machines. The associated optimal control problem has been well studied in the stochastic manufacturing literature; see [20, 21]. Let the system model be given as

$$
\frac{d X(t)}{d t}=(A u+B z)(t), \quad t \geq 0,
$$


where $X \in \mathbb{R}^{n}, u \in \mathbb{R}_{+}^{n}$, and $z \in \mathbb{R}_{+}$, and

$$
A=\left[\begin{array}{ccccc}
1 & -1 & 0 & \cdots & 0 \\
0 & 1 & -1 & \cdots & 0 \\
\vdots & & & \vdots & \\
0 & 0 & 0 & \cdots & 1
\end{array}\right], \quad B=\left[\begin{array}{c}
0 \\
\vdots \\
0 \\
-1
\end{array}\right]
$$

Here all upper subdiagonal entries in $A$ are -1 . The state space for $X$ is $\bar{Q} \triangleq$ $[0, \infty)^{n-1} \times(-\infty, \infty)$. Notice that the last component in $X$ is the inventory level of the final product, which may be negative and accordingly interpreted as backlog. The first $n-1$ entries in $X$ denote the buffer levels and hence are nonnegative. The variable $z$ denotes a finite state Markov chain describing the random demanding rate. The cost function to be minimized is of the form

$$
J(x, k, z, u)=E\left[\int_{0}^{\infty} e^{-\rho t} L(X(t), u(t)) d t \mid X(0)=x, k(0)=k, z(0)=z\right],
$$

where $(x, k, z)$ is the initial condition. Here $k(t) \in \mathbb{R}^{n}$ is vector Markov process with discrete values describing the machine capacity. Let the state space and generator for $(k, z)$ be denoted by $\mathcal{C} \times \mathcal{D}$ and $\Pi$, respectively. For the initial condition $(x, k, z)$, the admissible control set $\mathcal{U}_{x, k, z}$ consists of controls such that (i) $u(t)$ is adapted to $\mathcal{F}_{t}=\sigma(k(s), z(s), s \leq t)$, (ii) $0 \leq u(t) \leq k(t)$ (holding entrywise), and (iii) $X(t) \in \bar{Q}$ at all times $t \geq 0$. We also assume

$$
\left|L(x, u)-L\left(x^{\prime}, u^{\prime}\right)\right| \leq C\left(1+|x|^{d}+\left|x^{\prime}\right|^{d}\right)\left(\left|x-x^{\prime}\right|+\left|u-u^{\prime}\right|\right),
$$

where $d>0$ is a constant. For a given mode $k(t)=k=\left(k_{1}, \ldots, k_{n}\right) \in \mathcal{C}$, let the machine capacity region be denoted by $U_{k}=\left\{u=\left(u_{1}, \ldots, u_{n}\right)^{T} \mid 0 \leq u_{i} \leq k_{i}, i=\right.$ $1, \ldots, n\}$. Let $v(x, k, z)$ be the value function associated with the cost $J(x, k, z, u)$ and the admissible control set $\mathcal{U}_{x, k, z}$. The interested reader is referred to [20, Chapter 4] for a detailed account of this class of problems.

We write the HJB equation

$$
\rho v(x, k, z)=\inf _{u \in U_{k}}\left[v_{x}(x, k, z)(A u+B z)+[\Pi v(x, \cdot, \cdot)](k, z)+L(x, u)\right],
$$

where $(x, k, z) \in \bar{Q} \times \mathcal{C} \times \mathcal{D}$ and $\Pi v(x, \cdot, \cdot)$ is determined in an obvious manner. Set

$$
\tilde{H}\left(x, k, z, v_{x}(x, k, z), v(x, \cdot, \cdot), u\right)=v_{x}(x, k, z)(A u+B z)+[\Pi v(x, \cdot, \cdot)](k, z)+L(x, u) .
$$

Then (50) may be written in the compact form:

$$
\begin{aligned}
\rho v(x, k, z) & =\inf _{u \in U_{k}} \tilde{H}\left(x, k, z, v_{x}(x, k, z), v(x, \cdot, \cdot), u\right) \\
& \triangleq H\left(x, k, z, v_{x}(x, k, z), v(x, \cdot \cdot \cdot)\right), \quad(x, k, z) \in \bar{Q} \times \mathcal{C} \times \mathcal{D} .
\end{aligned}
$$

Now we apply the results in section 3 and characterize the value function as the unique constrained viscosity solution to (51).

THEOREM 12. The value function $v: \bar{Q} \times \mathcal{C} \times \mathcal{D} \rightarrow \mathbb{R}$ is the unique constrained viscosity solution to the HJB equation (51) in the function class $C_{p, L i p}^{\text {loc }}(\bar{Q} \times \mathcal{C} \times \mathcal{D})$.

Proof. The continuity and growth estimates have been given in [20, Chapter 4]. The viscosity sub/supersolution properties for $v$ under Definition 1 can be verified 
by a similar method as in proving Theorem 11, and uniqueness follows from Theorem 3.

It is worthwhile to note that within our solution notion, for $x$ on the boundary of $\bar{Q}$, the right-hand side of (50) is calculated by minimizing over $U_{k}$, and the state space constraint is not explicitly involved, which differs from [20, pp. 65-71] in dealing with state constraints. The key reason here is that the viscosity subsolution property is specified only on $Q$, and by use of this slightly weaker specification, we can still establish uniqueness on $\bar{Q}$ owing to the continuity of the solution.

8. Concluding remarks. In this paper we study optimal control of a class of stochastic hybrid systems with state space constraints. The notion of constrained viscosity solutions is introduced. We establish a comparison theorem for the subsolution and supersolution, and under some mild conditions for the general model, the value function is characterized as the unique constrained viscosity solution to the HJB equation. The uniqueness result obtained in the general setting is further applied to a communication buffer model and a standard manufacturing system.

For future research, it is of interest to generalize the state constrained viscosity solution analysis to systems with switch cost. To gain some motivation, we consider the fluid communication buffer model in section 6 . Intuitively, a high buffer level will produce a high holding cost, and on the other hand, a very low buffer level limits the controller in choosing a more beneficial action. Hence, with a certain combination of values for the buffer level $x$ and mode variable $(y, z)$, the control may switch rapidly between positive $u_{1}$ and positive $u_{2}$ in order to attain or approximate the optimal cost. This leads to the so-called chattering effect, which is undesirable in practical applications. We note that this kind of chattering may also occur in manufacturing systems where the machine's operation switches between the production of multiple products [20]. It is of interest to develop numerical methods to identify the critical buffer levels where chattering may occur. Furthermore, for chattering avoidance, an effective means is to introduce a switch cost, and then one needs to deal with quasi-variational inequalities [3, 4$]$ instead of a usual HJB equation. A detailed study of optimization and numerical computation of these hybrid systems with both state space constraints and switch cost will be reported in future work.

\section{Appendix. Proof of Theorem 11.}

It is obvious that $v \in C_{p, L i p}^{l o c}\left([0, \infty) \times S_{y} \times S_{z}\right)$. It suffices to show that $v$ is a constrained viscosity solution, and uniqueness follows from Theorem 3 since $C_{p, \text { Lip }}^{\text {loc }}\left([0, \infty) \times S_{y} \times S_{z}\right) \subset C_{p, H o l}^{l o c}\left([0, \infty) \times S_{y} \times S_{z}\right)$.

We give the proof by carrying out elementary estimates. Denote $\bar{Q}=[0, \infty)$ and $Q=(0, \infty)$. After suitably labeling, we may denote the joint process $(y(t), z(t))$ by an equivalent integer-valued Markov chain $\theta(t)$ with state space $\mathcal{P}=\{1,2, \ldots, m\}$ containing $m=m_{1} \times m_{2}$ entries, and let the associated generator for $\theta(s)$ be $\Pi_{\theta}=$ $\left(\pi_{i j}\right)_{m \times m}$. All of our estimates below may easily translate into a form in terms of the process $(y, z)$, and we omit the details. First, we show $v$ is a subsolution on $Q \times \mathcal{P}$. The functions $v(x, k), L(x, k, u)$, and $F(k, u), k \geq 1$ (instead of $v(x, i, j)$, etc.), are used in an obvious manner. For any given $k_{0} \in \mathcal{P}$, suppose $v\left(x, k_{0}\right)-\phi(x)$ attains a local maximum at $x_{0} \in Q$ in a neighborhood $N_{x_{0}} \subset Q$, where $\phi \in C^{1}(\bar{Q})$. Without loss of generality, we assume $v\left(x_{0}, k_{0}\right)=\phi\left(x_{0}\right)$, since otherwise $\phi(x)$ may be replaced by $\phi(x)-\phi\left(x_{0}\right)+v\left(x_{0}, k_{0}\right)$. It is easy to check that $v\left(x, k_{0}\right) \leq v\left(x_{0}, k_{0}\right)-\phi\left(x_{0}\right)+\phi(x)$ for all $x \in N_{x_{0}}$. 
For a given initial state $x_{0} \in Q$ and any $\bar{u} \in U$, there exist a sufficiently small interval $[0, \delta]$ and an admissible control $\tilde{u}$ defined on $[0, \infty)$ such that $\tilde{u}(t) \equiv \bar{u}$ on $[0, \delta]$ and $x(t) \in N_{x_{0}}$ for all $0<t \leq \delta$. For the given $x_{0}, \delta>0$ may be selected independently of the control. Let $\tau$ be the first jump time of $\theta(t)$ with initial state $k_{0} \in \mathcal{P}$. If $k_{0}$ is nonabsorbing, $\tau$ has an exponential probability density function $\left|\pi_{k_{0} k_{0}}\right| e^{\pi_{k_{0} k_{0}} t}, t \geq 0$. The estimates below are applicable to both nonabsorbing and absorbing $k_{0}$. By the dynamic programming principle, for $h \in(0, \delta)$, we have

$$
\begin{aligned}
\phi\left(x_{0}\right)= & v\left(x_{0}, k_{0}\right) \leq E \int_{0}^{h} e^{-\rho s} L(X, \theta, \tilde{u})(s) d s+E e^{-\rho h} v(X(h), \theta(h)) \\
\leq & E \int_{0}^{h} e^{-\rho s} L(X, \theta, \tilde{u})(s) d s+E e^{-\rho h} v(X(h), \theta(h)) 1_{(h<\tau)} \\
& +E e^{-\rho h} v(X(h), \theta(h)) 1_{(h \geq \tau)} \\
\leq & E \int_{0}^{h} e^{-\rho s} L(X, \theta, \tilde{u})(s) d s+E e^{-\rho h} v\left(x_{0}, k_{0}\right) 1_{(h<\tau)} \\
& +E e^{-\rho h}\left[\phi(X(h))-\phi\left(x_{0}\right)\right] 1_{(h<\tau)}+E e^{-\rho h} v(X(h), \theta(h)) 1_{(h \geq \tau)} \\
\triangleq & I_{1}+I_{2}+I_{3}+I_{4} .
\end{aligned}
$$

It is easy to obtain the estimates

$$
\begin{aligned}
I_{1}= & L\left(x_{0}, k_{0}, \bar{u}\right) h+o(h), \\
I_{2}= & {[1-\rho h+o(h)] v\left(x_{0}, k_{0}\right) e^{\pi_{k_{0} k_{0}} h} } \\
= & v\left(x_{0}, k_{0}\right)-\rho v\left(x_{0}, k_{0}\right) h+v\left(x_{0}, k_{0}\right) \pi_{k_{0} k_{0}} h+o(h), \\
I_{3}= & E e^{-\rho h}\left[\phi(X(h))-\phi\left(x_{0}\right)\right]-E e^{-\rho h}\left[\phi(X(h))-\phi\left(x_{0}\right)\right] 1_{(h \geq \tau)} \\
= & \phi_{x}\left(x_{0}\right) F\left(k_{0}, \bar{u}\right) h+o(h)+O\left(\left(E\left|\phi(X(h))-\phi\left(x_{0}\right)\right|^{2} \cdot E\left|1_{(h \geq \tau)}\right|^{2}\right)^{\frac{1}{2}}\right) \\
= & \phi_{x}\left(x_{0}\right) F\left(k_{0}, \bar{u}\right) h+o(h), \\
I_{4}= & \left.E e^{-\rho h} v\left(x_{0}, \theta(h)\right) 1_{(h \geq \tau)}+E e^{-\rho h}\left[v(X(h), \theta(h))-v\left(x_{0}, \theta(h)\right)\right] 1_{(h \geq \tau)}\right) \\
= & E e^{-\rho h} v\left(x_{0}, \theta(h)\right) 1_{(h \geq \tau)} \\
& +O\left(\left(E\left|v(X(h), \theta(h))-v\left(x_{0}, \theta(h)\right)\right|^{2} \cdot E\left|1_{(h \geq \tau)}\right|^{2}\right)^{\frac{1}{2}}\right) \\
= & E e^{-\rho h} v\left(x_{0}, \theta(h)\right) 1_{(h \geq \tau)}+o(h) \\
= & h \sum_{k \neq k_{0}} \pi_{k_{0} k} v\left(x_{0}, k\right)+o(h),
\end{aligned}
$$

where (A.2) is obtained by the continuity of $v$ with respect to $x$. Recalling $v\left(x_{0}, k_{0}\right)=$ $\phi\left(x_{0}\right)$, we get

$$
\begin{aligned}
0 \leq & I_{1}+I_{2}+I_{3}+I_{4}-\phi\left(x_{0}\right) \\
= & L\left(x_{0}, k_{0}, \bar{u}\right) h-\rho v\left(x_{0}, k_{0}\right) h+v\left(x_{0}, k_{0}\right) h \pi_{k_{0} k_{0}} \\
& +\phi_{x}\left(x_{0}\right) F\left(k_{0}, \bar{u}\right) h+h \sum_{k \neq k_{0}} \pi_{k_{0} k} v\left(x_{0}, k\right)+o(h) \\
= & -\rho v\left(x_{0}, k_{0}\right) h+\phi_{x}\left(x_{0}\right) F\left(k_{0}, \bar{u}\right) h+h \sum_{k \neq k_{0}} \pi_{k_{0} k}\left[v\left(x_{0}, k\right)-v\left(x_{0}, k_{0}\right)\right] \\
& +L\left(x_{0}, k_{0}, \bar{u}\right) h+o(h)
\end{aligned}
$$


since $\pi_{k_{0} k_{0}}+\sum_{k \neq k_{0}} \pi_{k_{0}, k}=0$. Letting $h \rightarrow 0$, we get the desired inequality for the viscosity subsolution since $\bar{u}$ is arbitrary.

Now we show $v$ is also a viscosity supersolution. Suppose there exists a neighborhood $N_{x_{0}}$ such that $v\left(x, k_{0}\right)-\phi(x)$ attains a local minimum at $x_{0} \in N_{x_{0}} \cap \bar{Q}$ for a given $k_{0} \in \mathcal{P}$; for any given $\varepsilon>0$, we can find a sequence of admissible controls $u^{(i)}$, $i \geq 1$, such that

$$
\begin{aligned}
v\left(x_{0}, k_{0}\right)+\frac{\varepsilon}{i} & \geq E \int_{0}^{\frac{1}{i}} e^{-\rho s} L\left(X, \theta, u^{(i)}\right)(s) d s+E e^{-\frac{\rho}{i}} v\left(X\left(\frac{1}{i}\right), \theta\left(\frac{1}{i}\right)\right) \\
& \triangleq I_{1}+I_{2}+I_{3}+I_{4}
\end{aligned}
$$

where we express the right-hand side by use of the same set of notation $I_{i}, 1 \leq i \leq 4$, as in (A.1) with $\tilde{u}$ replaced by $u^{(i)}$. Now we give the estimates as follows:

$$
\begin{aligned}
I_{1}+I_{3} & =E \int_{0}^{\frac{1}{i}} e^{-\rho s} L\left(x_{0}, k_{0}, u^{(i)}\right)(s) d s+E e^{-\frac{\rho}{i}}\left[\phi\left(X\left(\frac{1}{i}\right)\right)-\phi\left(x_{0}\right)\right]+o\left(\frac{1}{i}\right) \\
& =E \int_{0}^{\frac{1}{i}} L\left(x_{0}, k_{0}, u^{(i)}\right)(s) d s+E\left[\phi\left(X\left(\frac{1}{i}\right)\right)-\phi\left(x_{0}\right)\right]+o\left(\frac{1}{i}\right) \\
& =E \int_{0}^{\frac{1}{i}}\left[L\left(x_{0}, k_{0}, u^{(i)}\right)+\phi_{x}(X) F\left(k_{0}, u^{(i)}\right)\right](s) d s+o\left(\frac{1}{i}\right) \\
& =E \int_{0}^{\frac{1}{i}}\left[L\left(x_{0}, k_{0}, u^{(i)}\right)+\phi_{x}\left(x_{0}\right) F\left(k_{0}, u^{(i)}\right)\right](s) d s+o\left(\frac{1}{i}\right) \\
& \geq \frac{1}{i} \inf _{u \in U}\left[L\left(x_{0}, k_{0}, u^{(i)}\right)+\phi_{x}\left(x_{0}\right) F\left(k_{0}, u^{(i)}\right)\right]+o\left(\frac{1}{i}\right) \\
I_{2}+I_{4} & =v\left(x_{0}, k_{0}\right)-\frac{\rho}{i} v\left(x_{0}, k_{0}\right)+\frac{1}{i} \sum_{k \neq k_{0}} \pi_{k_{0} k}\left[v\left(x_{0}, k\right)-v\left(x_{0}, k_{0}\right)\right]+o\left(\frac{1}{i}\right),
\end{aligned}
$$

where the higher order term $o\left(\frac{1}{i}\right)$ is derived via basic estimates using the dynamics of $X(t)$ and the Markov chain $\theta(t)$ and holds uniformly with respect to $\varepsilon$. Taking $i \rightarrow \infty$, it follows from (A.3)-(A.5) that

$$
\rho v\left(x_{0}, k_{0}\right)+\varepsilon \geq \inf _{u \in U}\left\{\phi_{x}\left(x_{0}\right) F\left(k_{0}, \bar{u}\right)+\sum_{k \neq k_{0}} \pi_{k_{0} k}\left[v\left(x_{0}, k\right)-v\left(x_{0}, k_{0}\right)\right]+L\left(x_{0}, k_{0}, \bar{u}\right)\right\} .
$$

Since $\varepsilon>0$ is arbitrary, it follows that $v$ is a viscosity supersolution on $\bar{Q} \times \mathcal{P}$.

Acknowledgments. I thank the referees and the Associate Editor for their valuable comments and suggestions, which have helped improve the quality of the paper, and thank the Corresponding Editor Professor T. E. Duncan for his efforts in handling the review process. The support of Professor R. J. Evans, Dr. S. Dey, and Dr. G. N. Nair of Department of Electrical and Electronic Engineering, The University of Melbourne, is gratefully acknowledged.

\section{REFERENCES}

[1] F. BAGAgiolo AND M. BARDI, Singular perturbation of a finite horizon problem with state-space constraints, SIAM J. Control Optim., 36 (1998), pp. 2040-2060.

[2] M. Bardi and I. Capuzzo-Dolcetta, Optimal Control and Viscosity Solutions of HamiltonJacobi-Bellman Equations, Birkhäuser, Boston, 1997. 
[3] A. Bensoussan and J. L. Lions, Impulse Control and Quasi-Variational Inequalities, Bordes, Paris, 1984.

[4] I. Capuzzo-Dolcetta and L. C. Evans, Optimal switching for ordinary differential equations, SIAM J. Control Optim., 22 (1984), pp. 143-161.

[5] I. Capuzzo-Dolcetta And P.-L. Lions, Hamilton-Jacobi equations with state constraints, Trans. Amer. Math. Soc., 318 (1990), pp. 643-683.

[6] M. H. A. Davis, Markov Models and Optimization, Chapman and Hall/CRC, London, 1993.

[7] A. I. Elwalid AND D. Mitra, Effective bandwidth of general Markovian traffic sources and admission control of high speed networks, IEEE/ACM Trans. Networking, 1 (1993), pp. 329343.

[8] W. H. Fleming and Q. Zhang, Risk-sensitive production planning of a stochastic manufacturing system, SIAM J. Control Optim., 36 (1998), pp. 1147-1170.

[9] J. P. Hespanha, Stochastic hybrid systems: application to communication networks, in Hybrid Systems: Computation and Control, R. Alur and G. J. Pappas, eds., Lect. Notes Comput. Sci. 2993, Springer, Berlin, 2004, pp. 387-401.

[10] M. Huang AND S. DeY, Joint rate/power adaptation and dynamic buffer management in wireless data relay networks, in Proceedings of the American Control Conference, Minneapolis, MN, 2006, pp. 6097-6102.

[11] H. IsHII, Uniqueness of unbounded viscosity solutions of Hamilton-Jacobi equations, Indiana Univ. Math. J., 33 (1984), pp. 721-748.

[12] H. Ishil And S. Kolke, A new formulation of state constraint problems for first-order PDEs, SIAM J. Control Optim., 34 (1996), pp. 554-571.

[13] H. Ishi And P. LORETI, A class of stochastic optimal control problems with state constraint, Indiana Univ. Math. J., 51 (2002), pp. 1167-1196.

[14] Y. Ji And H. J. Chizeck, Controllability, stabilizability, and continuous-time Markovian jump linear quadratic control, IEEE Trans. Automat. Control, 35 (1990), pp. 777-788.

[15] P. LoRETI, Some properties of constrained viscosity solutions of Hamilton-Jacobi-Bellman equations, SIAM J. Control Optim., 25 (1987), pp. 1244-1252.

[16] P. Loreti And M. E. Tessitore, Approximation and regularity results on constrained viscosity solutions of Hamilton-Jacobi-Bellman equations, J. Math. Syst. Estim. Control, 4 (1994), pp. 467-483.

[17] D. MitrA, Stochastic theory of a fluid model of multiple failure-susceptible producers and consumers coupled by a buffer, Adv. Appl. Prob., 20 (1988), pp. 646-676.

[18] M. МоттA, On nonlinear optimal control problems with state constraints, SIAM J. Control Optim., 33 (1995), pp. 1411-1424.

[19] S. Rajagopal, V. G. Kulkarni, and S. Stidham, Jr., Optimal flow control of a stochastic fluid-flow system, IEEE J. Sel. Areas Comm., 13 (1995), pp. 1219-1228.

[20] S. P. Sethi And Q. Zhang, Hierarchical Decision Making in Stochastic Manufacturing Systems, Birkhäuser, Boston, 1994.

[21] S. P. Sethi And X. Y. Zhou, Stochastic dynamic job shops and hierarchical production planning, IEEE Trans. Automat. Control, 39 (1994), pp. 2061-2076.

[22] H. M. Soner, Optimal control with state-space constraint I, SIAM J. Control Optim., 24 (1986), pp. 552-561.

[23] H. M. Soner, Optimal control with state-space constraint II, SIAM J. Control Optim., 24 (1986), pp. 1110-1122.

[24] D. D. Sworder, Feedback control of a class of linear systems with jump parameters, IEEE Trans. Automat. Control, 14 (1969), pp. 9-14.

[25] M. H. Veatch AND L. M. Wein, Optimal control of a two-station tandem production/inventory system, Oper. Res., 42 (1994), pp. 337-350.

[26] M. XIAO AND T. BAŞAR, Optimal control of piecewise deterministic nonlinear systems with controlled transitions: viscosity solutions, their existence and uniqueness, in Proceedings of the 38th IEEE Conference on Decision and Control, Phoenix, AZ, 1999, pp. 4712-4717.

[27] M. XIAO AND T. BAŞAR, Viscosity solutions of two classes of coupled HJB equations, J. Inequal. Appl., 6 (2001), pp. 519-545.

[28] M. W. Wonham, Random differential equations in control theory, in Probabilistic Methods in Applied Mathematics, Vol II, A.T. Bharucha, ed., Academic Press, New York, 1971, pp. 131-213. 